\title{
GRB 980425 host: [C II], [O I], and CO lines reveal recent enhancement of star formation due to atomic gas inflow ${ }^{\star}$
}

\author{
M. J. Michałowski ${ }^{1}$, J. M. Castro Cerón ${ }^{2}$, J. L. Wardlow ${ }^{3,4}$, A. Karska ${ }^{5,6}$, H. Messias ${ }^{7}$, P. van der Werf ${ }^{8}$, L. K. Hunt ${ }^{9}$, \\ M. Baes ${ }^{10}$, A. J. Castro-Tirado ${ }^{11}$, G. Gentile ${ }^{10,12}$, J. Hjorth ${ }^{3}$, E. Le Floc'h ${ }^{13}$, R. Pérez-Martínez ${ }^{14}$, \\ A. Nicuesa Guelbenzu ${ }^{15}$, J. Rasmussen ${ }^{3,16}$, J. R. Rizzo ${ }^{17}$, A. Rossi ${ }^{18}$, M. Sánchez-Portal ${ }^{2}$, P. Schady ${ }^{19}$, \\ J. Sollerman ${ }^{20}$, and D. $\mathrm{Xu}^{21}$ \\ (Affiliations can be found after the references)
}

Received 30 July 2016 / Accepted 6 September 2016

\begin{abstract}
Context. Accretion of gas from the intergalactic medium is required to fuel star formation in galaxies. We have recently suggested that this process can be studied using host galaxies of gamma-ray bursts (GRBs).

Aims. Our aim is to test this possibility by studying in detail the properties of gas in the closest galaxy hosting a GRB (980425).

Methods. We obtained the first ever far-infrared (FIR) line observations of a GRB host, namely Herschel/PACS resolved [C II] $158 \mu \mathrm{m}$ and [OI] $63 \mu \mathrm{m}$ spectroscopy, and an APEX/SHeFI CO(2-1) line detection and ALMA CO(1-0) observations of the GRB 980425 host.

Results. The GRB 980425 host has elevated [C II]/FIR and [O I]/FIR ratios and higher values of star formation rates (SFR) derived from line ([C II], [OI], $\mathrm{H} \alpha$ ) than from continuum (UV, IR, radio) indicators. [C II] emission exhibits a normal morphology, peaking at the galaxy centre, whereas $[\mathrm{OI}]$ is concentrated close to the GRB position and the nearby Wolf-Rayet region. The high [OI] flux indicates that there is high radiation field and high gas density at these positions, as derived from modelling of photo-dissociation regions. The [C II]/CO luminosity ratio of the GRB 980425 host is close to the highest values found for local star-forming galaxies. Indeed, its CO-derived molecular gas mass is low given its SFR and metallicity, but the [C II]-derived molecular gas mass is close to the expected value.

Conclusions. The [OI] and H I concentrations and the high radiation field and density close to the GRB position are consistent with the hypothesis of a very recent (at most a few tens of Myr ago) inflow of atomic gas triggering star formation. In this scenario dust has not had time to build up (explaining high line-to-continuum ratios). Such a recent enhancement of star formation activity would indeed manifest itself in high $S F R_{\text {line }} / S F R_{\text {continum }}$ ratios because the line indicators are sensitive only to recent ( $\lesssim 10 \mathrm{Myr}$ ) activity, whereas the continuum indicators measure the SFR averaged over much longer periods $(\sim 100 \mathrm{Myr})$. Within a sample of 32 other GRB hosts, 20 exhibit $S F R_{\text {line }} / S F R_{\text {continuum }}>1$ with a mean ratio of $1.74 \pm 0.32$. This is consistent with a very recent enhancement of star formation that is common among GRB hosts, so galaxies that have recently experienced inflow of gas may preferentially host stars exploding as GRBs. Therefore GRBs may be used to select a unique sample of galaxies that is suitable for the investigation of recent gas accretion.
\end{abstract}

Key words. dust, extinction - galaxies: individual: ESO 184-G82 - galaxies: ISM - galaxies: star formation - submillimeter: galaxies gamma-ray burst: individual: 980425

\section{Introduction}

One of the most important aspects of the evolution of the Universe is how galaxies acquire the gas that fuels star formation. Numerical galaxy formation models require significant gas inflows from the intergalactic medium (IGM) to fuel star formation (e.g. Schaye et al. 2010), and indeed the current gas reservoirs in many galaxies are too low to sustain the current level of star formation, even for normal galaxies like the Milky Way (e.g. Draine 2009). However, despite much indirect evidence for gas inflows (e.g. Sancisi et al. 2008; Sánchez Almeida et al. 2013, 2014b,a; Stott et al. 2013; Wang et al. 2015), they have been claimed to be observationally detected in only a handful of galaxies (Ribaudo et al. 2011; Martin et al. 2014; Michałowski et al. 2015; Turner et al. 2015; Rauch et al. 2016), including host galaxies of long (duration $>2 \mathrm{~s}$ ) gamma-ray bursts (GRBs).

Because GRBs are explosions of very massive and shortlived stars (e.g. Hjorth et al. 2003; Stanek et al. 2003; for a review see Hjorth \& Bloom 2012), they pinpoint locations of

\footnotetext{
* Herschel is an ESA space observatory with science instruments provided by European-led Principal Investigator consortia and with important participation from NASA.
}

recent star formation. Star formation is usually assumed to be fuelled by molecular gas (Carilli \& Walter 2013; Rafelski et al. 2016), but several GRB host galaxies show a deficit in molecular gas $\left(\mathrm{H}_{2}\right.$; Hatsukade et al. 2014; Stanway et al. 2015b). This deficit is unusual for galaxies with normal star formation rates (SFRs) unlike for extreme starbursts. This deficiency is not due to a high CO-to- $\mathrm{H}_{2}$ conversion factor (which happens at low metallicity; Bolatto et al. 2013), as CO-targeted GRB hosts have metallicities $12+\log (\mathrm{O} / \mathrm{H}) \sim$ 8.7-9.0 (Castro-Tirado et al. 2007; Levesque et al. 2010b; Stanway et al. 2015a), using the calibrations of Pagel et al. (1979), Kewley \& Dopita (2002), Pettini \& Pagel (2004), and Maiolino et al. (2008), which is close to the solar metallicity of $12+\log (\mathrm{O} / \mathrm{H}) \sim 8.66$ (Asplund et al. 2004). Moreover, optical spectroscopy of GRB afterglows implies that the molecular phase constitutes only a small fraction of the gas along the GRB line of sight (Vreeswijk et al. 2004; Fynbo et al. 2006; Tumlinson et al. 2007; Prochaska et al. 2009; D'Elia et al. 2010, 2014; Krühler et al. 2013; Friis et al. 2015).

On the other hand, the Australia Telescope Compact Array (ATCA) $21 \mathrm{~cm}$ line survey of GRB host galaxies revealed high levels of atomic hydrogen $(\mathrm{HI})$, suggesting that the connection 
between atomic gas and star formation is stronger than previously thought (Michałowski et al. 2015). Star formation may be directly fuelled by atomic gas, as has been theoretically shown to be possible (Glover \& Clark 2012; Krumholz 2012; Hu et al. 2016), and this is supported by the existence of H I-dominated, star-forming regions in other galaxies (Bigiel et al. 2008, 2010; Fumagalli \& Gavazzi 2008; Elmegreen et al. 2016). This can happen in a low metallicity gas that is recently acquired, even if the metallicity in other parts of a galaxy is higher, near the onset of star formation because cooling of gas (necessary for star formation) is faster than the H I-to- $\mathrm{H}_{2}$ conversion (Krumholz 2012). Indeed, large atomic gas reservoirs, together with low molecular gas masses (Hatsukade et al. 2014; Stanway et al. 2015b) and stellar masses (Perley et al. 2013, 2015; Vergani et al. 2015), indicate that GRB hosts are preferentially galaxies that have very recently started a star formation episode. This provides a natural route for forming GRBs in low-metallicity environments, as found for most GRB hosts (Fruchter et al. 2006; Modjaz et al. 2008; Levesque et al. 2010a; Han et al. 2010; Boissier et al. 2013; Schulze et al. 2015; Vergani et al. 2015; Japelj et al. 2016; Perley et al. 2016), except of a few examples of hosts with solar or super-solar metallicities (Prochaska et al. 2009; Levesque et al. 2010b; Krühler et al. 2012; Savaglio et al. 2012; Elliott et al. 2013; Schulze et al. 2014; Hashimoto et al. 2015; Schady et al. 2015; Stanway et al. 2015a). Indeed, the GRB collapsar model requires that most of the GRB progenitors have low metallicity (below solar) in order to reduce the loss of mass and angular momentum that is required for launching the jet (Yoon \& Langer 2005; Yoon et al. 2006; Woosley \& Heger 2006). We note however that other models, while still predicting the metallicity preference (e.g. Izzard et al. 2004; Podsiadlowski et al. 2004; Detmers et al. 2008), allow higher metallicities owing to differential rotation (Georgy et al. 2012), binary evolution (Podsiadlowski et al. 2010; van den Heuvel \& Portegies Zwart 2013), or weaker magnetic fields (Petrovic et al. 2005).

Summarising the ATCA H I data support a scenario whereby GRBs are preferentially produced when low-metallicity gas accretes onto a galaxy and undergoes rapid cooling and star formation before it either forms $\mathrm{H}_{2}$ or mixes with the higher metallicity gas in the remainder of the galaxy. This scenario provides a natural explanation for the low-metallicity and low- $M_{\mathrm{H}_{2}}$ preferences. In contrast, at later stages of star formation molecular gas is the dominant phase in the interstellar medium, but the metals are well mixed, and gas has been further enriched, so massive stars do not end their lives as GRBs, and such metal- and molecularrich galaxies do not become GRB hosts.

The gas inflow scenario is also supported by the existence of the companion H I object with no optical counterpart $\sim 19 \mathrm{kpc}$ from the GRB 060505 host, which may be a stream of gas inflowing on this galaxy. In addition the HI centroids of the GRB 980425 and 060505 hosts do not coincide with the optical centres of these galaxies, but are located close to the GRB positions (Michałowski et al. 2015). The concentration of $\mathrm{HI}$ close to the GRB 980425 position has been confirmed with highresolution $\mathrm{H}$ I imaging by the Giant Metrewave Radio Telescope (GMRT; Arabsalmani et al. 2015).

Here, we test the gas inflow scenario by investigating in detail the properties of gas in the GRB 980425 host. GRB 980425 at redshift $z=0.0085$ (Tinney et al. 1998) and its associated supernova SN 1998bw (Galama et al. 1998) is the closest GRB, and is located in the barred spiral galaxy ESO 184-G82. Hence, it is one of the few GRB hosts for which resolved gas properties can be studied. Of particular importance is a

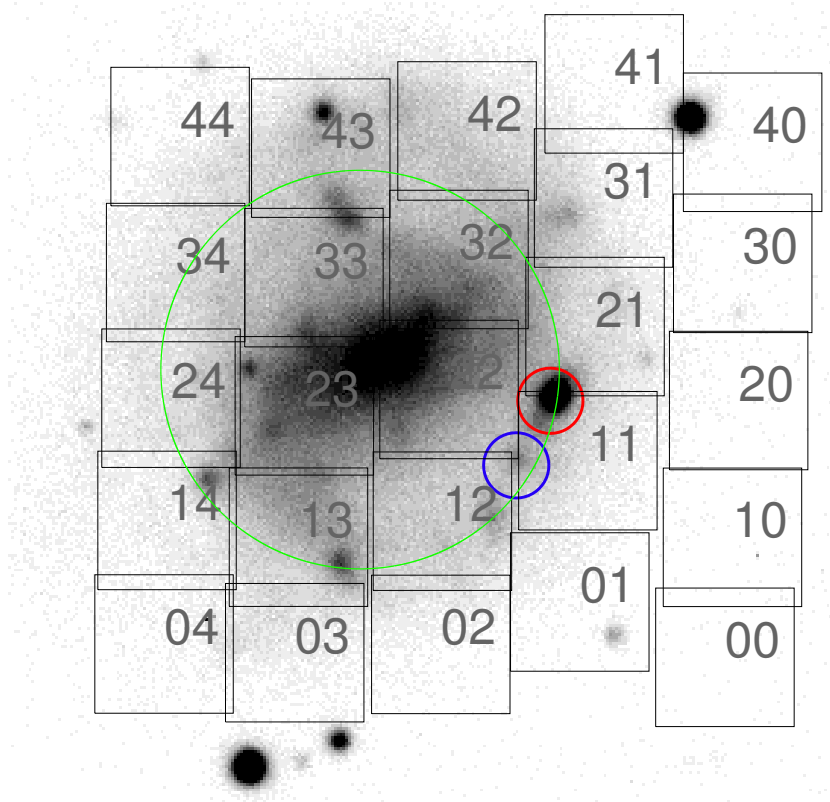

Fig. 1. Image in the $R$-band of the GRB 980425 host (Sollerman et al. $2005)$ with the position and numbers of the PACS spaxels indicated. The panel is $60^{\prime \prime} \times 60^{\prime \prime}(11 \mathrm{kpc} \times 11 \mathrm{kpc})$. The blue and red circles show the position of the GRB and WR region, respectively. Green circle shows the APEX beam for the $\mathrm{CO}(2-1)$ observations.

Wolf-Rayet (WR) region $\sim 800$ pc northwest of the GRB/SN position (Hammer et al. 2006), dominating the emission of the galaxy at $24 \mu \mathrm{m}$ (Le Floc'h et al. 2006), $100 \mu \mathrm{m}$, and radio (Michałowski et al. 2014b). This region is young (1-6 Myr; Hammer et al. 2006; Christensen et al. 2008) and exhibits the lowest metallicity among star-forming regions within the host with $12+\log (\mathrm{O} / \mathrm{H})=8.16$, i.e. 0.3 solar (Christensen et al. 2008), compared with $12+\log (\mathrm{O} / \mathrm{H})=8.6$, i.e. 0.8 solar (Sollerman et al. 2005) for the entire host. These values were obtained with the calibrations of Pettini \& Pagel (2004) and Kewley \& Dopita (2002), respectively.

We use a cosmological model with $H_{0}=70 \mathrm{~km} \mathrm{~s}^{-1} \mathrm{Mpc}^{-1}$, $\Omega_{\Lambda}=0.7$, and $\Omega_{\mathrm{m}}=0.3$, and so GRB 980425 at $z=0.0085$ is at a luminosity distance of $36.5 \mathrm{Mpc}$ and $1^{\prime \prime}$ corresponds to $175 \mathrm{pc}$ at its redshift.

\section{Data}

We obtained Herschel (Pilbratt et al. 2010) observations of the GRB 980425 host (project No. OT2_jmcastro_3, PI: J. M. Castro Cerón) using the Photodetector Array Camera and Spectrometer (PACS; Poglitsch et al. 2010) with a total integration time of $4097 \mathrm{~s}$, on $20 \mathrm{Apr} .2013^{1}$. The data were taken in the line spectroscopy mode with medium chopping/nodding to off source (and off-galaxy) positions, which aids background subtraction. Because of the expected relative brightnesses of the [O I] $63 \mu \mathrm{m}$ and [C II] $158 \mu \mathrm{m}$ lines, the [O I] wavelength was observed approximately nine times longer. The positions of the spaxels are shown on the optical image of the host in Fig. 1.

Data reduction for PACS was performed in the Herschel Imaging Processing Environment (HIPE; Ott 2010) v12.1.0 with version 65.0 of the PACS calibration tree. We used the IPIPE background normalisation script for chop/nod range scan data,

1 OBSIDs: 1342270641. 
which is optimised for faint sources by using off-source positions in the background subtraction and flux calibration. To avoid introducing correlated noise we set the upsample factor to 1 during flat fielding, and to minimise signal losses we also masked the spectral regions in which spectral lines are expected during this process. The final spectra are binned to be Nyquist sampled at the native PACS resolution.

We also performed $\mathrm{CO}(2-1)$ observations of the GRB 980425 host on 29 Aug. (precipitable water vapour [pwv] of $1.7 \mathrm{~mm}$ ), $12 \mathrm{Sep}$. (pwv of $0.75-0.85 \mathrm{~mm}$ ), 16 Sep. (pwv of $1.43-1.57 \mathrm{~mm}$ ), 31 Oct. (pwv of $1.22-1.96 \mathrm{~mm}$ ), and 01 Nov. 2015 (pwv of $0.66-0.85 \mathrm{~mm}$ ) using the Swedish Heterodyne Facility Instrument (SHeFI; Vassilev et al. 2008) mounted at the Atacama Pathfinder Experiment (APEX; Güsten et al. 2006; project Nos. 096.D-0280 and 096.F-9302, PI: M. Michałowski). A total of $4.92 \mathrm{~h}$ of on-source data were obtained. The APEX-1 single-sideband (SSB) was tuned to the observed frequency of the $\mathrm{CO}(2-1)$ line of $228.6 \mathrm{GHz}$. At this frequency the APEX beam size is $27^{\prime \prime}(\sim 4.8 \mathrm{kpc}$ at the distance of the GRB). All observations were completed in the on-off pattern and the position-switching mode. The fluxes were corrected using the main beam efficiency of 0.75 . We reduced and analysed the data using the Continuum and Line Analysis Single Dish Software (CLASS) package within the Grenoble Image and Line Data Analysis Software ${ }^{2}$ (GILDAS; Pety 2005).

We performed Band 3 ALMA observations on 1 Sep. 2012 (project No. 2011.0.00046.S, PI: M. Michałowski). A total of $67.4 \mathrm{~min}$ of on-source data were obtained. Four $1.875 \mathrm{GHz}$ spectral windows were centred at 100.6, 102.4, 112.5, and $114.3 \mathrm{GHz}$. Twenty-three antennas and baselines ranging between 24 and $384 \mathrm{~m}$ were available. Neptune, J1733-130, and J1945-552 were used as flux, bandpass, and phase calibrators, respectively. The amount of precipitable water vapour ranged between $1.8-2.15 \mathrm{~mm}$. The data reduction and analysis were carried out using the CASA package (McMullin et al. 2007). The original spectral resolution was $\sim 488 \mathrm{kHz}\left(\sim 1.3 \mathrm{~km} \mathrm{~s}^{-1}\right)$. The continuum map was presented in Michałowski et al. (2014b), whereas here we present a data cube at the frequency of the $\mathrm{CO}(1-0)$ line $(114.288 \mathrm{GHz})$ binning ten channels resulting in spectral resolution of $\sim 4.9 \mathrm{MHz}$ (corresponding to $\sim 13 \mathrm{~km} \mathrm{~s}^{-1}$ ). The synthesised beam of the cube is $\sim 1.7^{\prime \prime}$.

\section{Methods}

We obtained three estimates of SFRs. First, we calculated $100 \mu \mathrm{m}$ fluxes within each spaxel using the Herschel/PACS map presented in Michałowski et al. (2014b). We converted these fluxes to total (8-1000 micron) infrared (IR) luminosities using the spectral energy distribution (SED) model of the WR region in the GRB 980425 host (Michałowski et al. 2014b), and to SFRs using the Kennicutt (1998) conversion for the Chabrier (2003) initial mass function (IMF; $S F R / M_{\odot} \mathrm{yr}^{-1}=10^{-10} L_{\mathrm{IR}} / L_{\odot}$ ). We probe close to the peak of the SED, so if we used other templates (e.g. Silva et al. 1998; Iglesias-Páramo et al. 2007; Michałowski et al. 2008, 2010a,b) we would obtain similar results. Then, we estimated the SFRs from the [C II] and [O I] lines using the conversion of De Looze et al. (2014, their Table 3, the first two rows).

Using far-infrared (FIR) luminosities integrated over 40$120 \mu \mathrm{m}$, we modelled the $[\mathrm{OI}] /[\mathrm{C} \mathrm{II}]$ and $([\mathrm{OI}]+[\mathrm{CII}]) /$ FIR ratios using the Photo Dissociation Region (PDR) Toolbox

\footnotetext{
2 http://www. iram. fr/IRAMFR/GILDAS
}

(Pound \& Wolfire 2008; Kaufman et al. 2006) ${ }^{3}$ with the addition of our own routine to extract errors on density and radiation field intensity from two-dimensional $\chi^{2}$ distributions. In this way we derived the gas density, $n$, and the ultraviolet (UV) strength of the radiation field, $G_{0}$, in the unit of the radiation field strength in the solar neighbourhood of $1.6 \times 10^{-3} \mathrm{erg} \mathrm{s}^{-1} \mathrm{~cm}^{-2}$ (or Habing unit; Habing 1968).

We estimated the molecular gas mass from the $\mathrm{CO}(2-1)$ line assuming the flux conversion $S_{\mathrm{CO}(1-0)}=0.5 \times S_{\mathrm{CO}(2-1)}$ (Fig. 4 in Carilli \& Walter 2013), i.e. $L_{\mathrm{CO}(1-0)}^{\prime}=2 \times L_{\mathrm{CO}(2-1)}^{\prime}$, assuming the Galactic CO-to- $\mathrm{H}_{2}$ conversion factor $\alpha_{\mathrm{CO}}=$ $5 M_{\odot} /\left(\mathrm{K} \mathrm{km} \mathrm{s}^{-1} \mathrm{pc}^{2}\right)$.

Finally, we estimated the molecular gas mass from the $[\mathrm{C} \mathrm{II}]$ line using the $[\mathrm{C}$ II $] / \mathrm{CO}(1-0)$ luminosity ratio of around 5000, typical for star-forming galaxies (Crawford et al. 1985; Wolfire et al. 1989; Stacey et al. 1991, 2010; Swinbank et al. 2012; Rigopoulou et al. 2014; Gullberg et al. 2015; Hughes et al. 2016). As noted by Neri et al. (2014), the luminosities of these lines expressed in brightness temperature units, $L^{\prime}\left(\mathrm{K} \mathrm{km} \mathrm{s}^{-1} \mathrm{pc}^{2}\right)$, are approximately equal (within a factor of two). Hence, we substituted $L_{\mathrm{CO}(1-0)}^{\prime}$ with $L_{[\mathrm{C} \mathrm{II}]}^{\prime}$ and obtained molecular gas masses with $M_{\mathrm{H}_{2}}=\alpha_{\mathrm{CO}} L_{[\mathrm{C} \mathrm{II}}^{\prime}$, assuming the same $\mathrm{CO}$-to- $\mathrm{H}_{2}$ conversion factor as above.

\section{Results}

The resulting [C II] and [OI] spectra in each PACS spaxel are presented in the top row of Fig. 2, whereas the summed spectra of the entire galaxy are shown in Fig. 3. The integrated fluxes were measured within inner dotted lines, with the errors within the outer lines excluding the inner region. Fluxes and luminosities are presented in Table A.1. The flux spatial distributions are shown in the middle row of Fig. 2. The [C II] flux distribution follows a normal radial dependence with the brightest spaxel at the centre of the galaxy. In contrast, most of the [O I] flux is not at the galaxy centre, but is concentrated close to the WR region (spaxel 11).

The ratios of the [C II] and [OI] lines and the lineto-continuum ratios of all spaxels and the entire host are shown in Fig. 4, and compared with local normal star-forming galaxies (Malhotra et al. 2001), dwarf galaxies (Cormier et al. 2015) from the Dwarf Galaxy Survey (Madden et al. 2013) and the Large Magellanic Cloud (LMC; Poglitsch et al. 1995; Israel et al. 1996). The GRB 980425 host has elevated [C II]/FIR and $[\mathrm{OI}] / \mathrm{FIR}$ ratios for its FIR luminosity and a low $[\mathrm{O} \mathrm{I}] /[\mathrm{C} \mathrm{II}]$ ratio.

The results of the PDR modelling are shown in the bottom row of Fig. 2 and in Fig. 5. The derived densities and radiation field strengths together with SFRs and molecular gas masses are presented in Table 1. Spaxel 11 has the highest [O I]/[C II] luminosity ratio $(\sim 1.7)$ among all regions in the host, which leads to the highest radiation field of $G_{0} \sim 1000$. Spaxels near the WR regions in the southwestern part of the galaxy (number 11, 22, 31) also have the highest densities in excess of $1000 \mathrm{~cm}^{-3}$.

Figure 6 shows the SFRs derived using luminosities of emission lines: [C II], [O I] (the calibration of De Looze et al. 2014) and $\mathrm{H} \alpha$ (as reported by Sollerman et al. 2005; Christensen et al. $2008)$ as a function of SFRs derived from total IR $(8-1000 \mu \mathrm{m})$ continuum emission. All line estimates are $\sim 1.5-6$ times larger than the IR and UV estimates (see Michałowski et al. 2009, $2014 b$ ). This is robust because we are comparing the line

\footnotetext{
dustem. astro.umd. edu/pdrt
} 

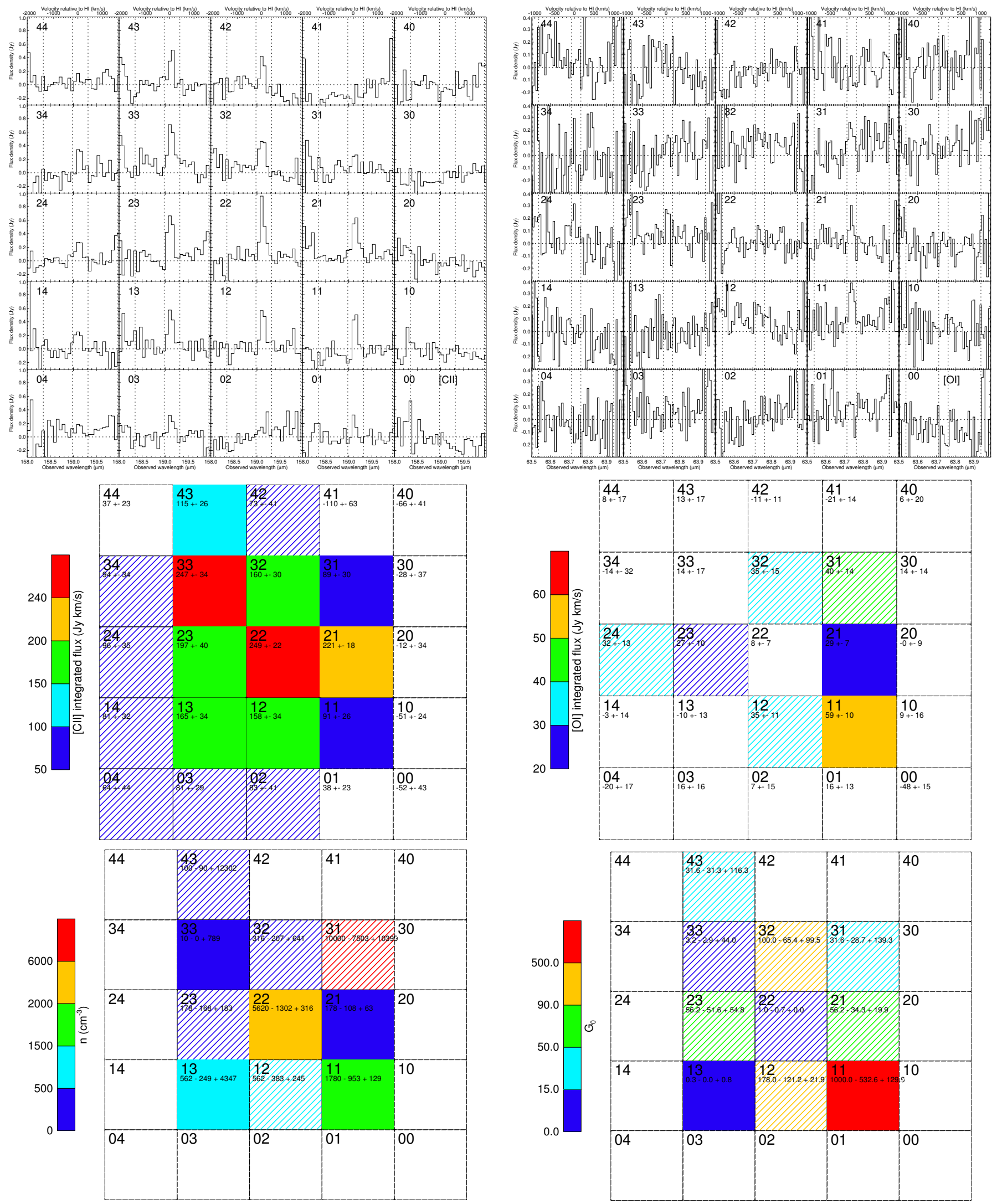

Fig. 2. Top: [C II] (left) and [O I] (right) spectra of each spaxel. The integrated fluxes were measured within inner dotted lines, with the errors within the outer lines excluding the inner region. Middle: [C II] (left) and [O I] (right) spatial distribution of integrated flux in each spaxel. Bottom: distribution of derived density and radiation field strength. In order to improve the readability of the plots we adopted the following convention. The line-filled boxes correspond to upper limits on the corresponding property if the flux measurement has $<3 \sigma$ significance, but if the measured value is below the lowest value on the colour bar, then the box is white. If the property cannot be measured ( $n$ and $G_{0}$ for spaxels with no detections at $[\mathrm{C} \mathrm{II}]$ and $[\mathrm{O} \mathrm{I}])$, then the box is also white with no values indicated. The [C II] emission exhibits a normal radial profile, whereas [O I] emission is concentrated close to the WR region. 

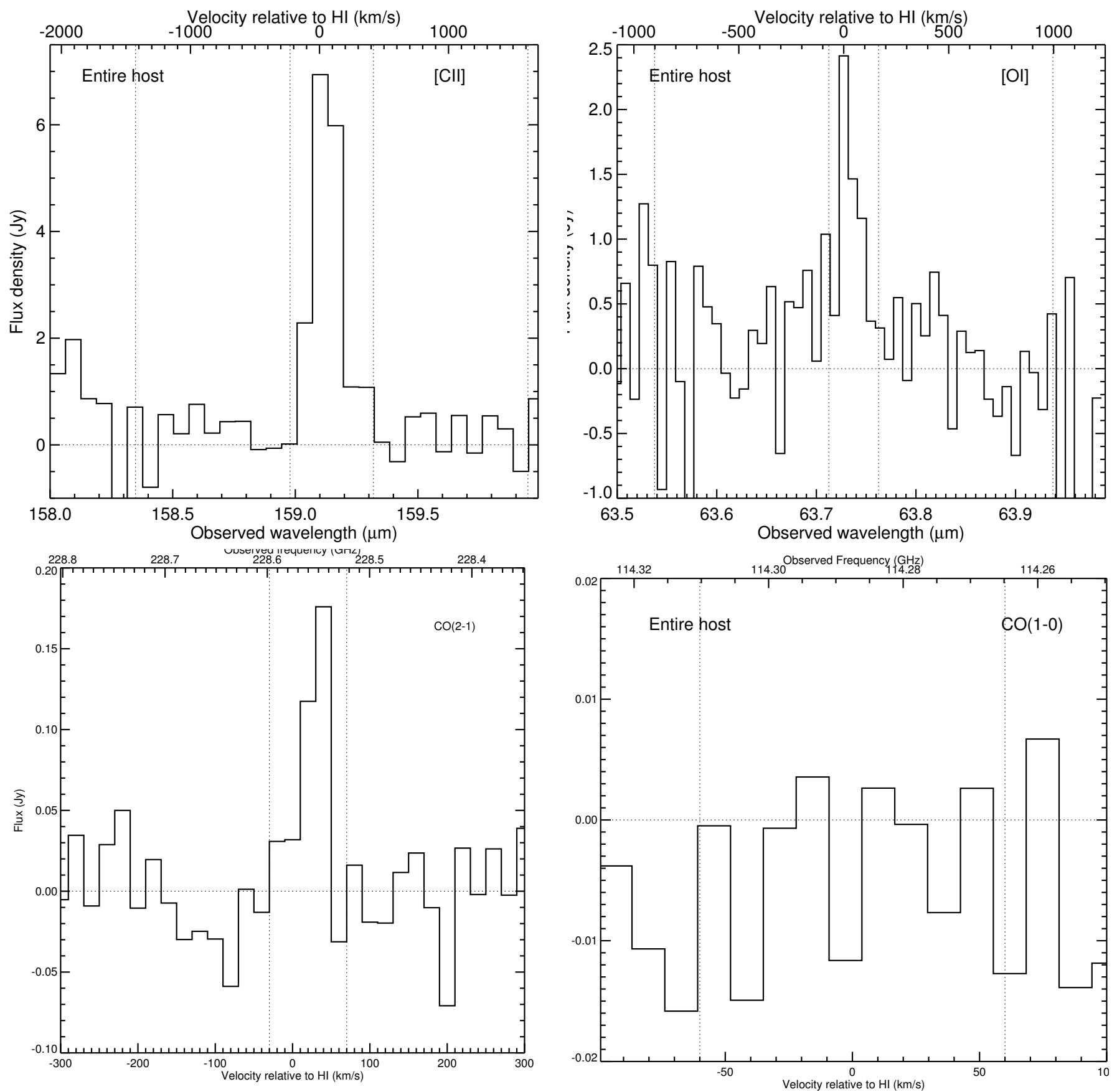

Fig. 3. [C II] (top left), [O I] (top right), $\mathrm{CO}(2-1)$ (bottom left), and $\mathrm{CO}(1-0)$ (bottom right) spectra of the entire host. The integrated fluxes were measured within inner dotted lines, with the errors within the outer lines excluding the inner region.

estimates to the relatively high value of $0.26 M_{\odot} \mathrm{yr}^{-1}$ obtained by SED modelling in Michałowski et al. (2014b). If we used the calibration of Kennicutt (1998), then we would obtain $0.1 M_{\odot} \mathrm{yr}^{-1}$, whereas that of Murphy et al. (2011, their Eq. (18)) would give $0.17 M_{\odot} \mathrm{yr}^{-1}$. On the other hand, if, instead of the calibration of De Looze et al. (2014), we used that of Sargsyan et al. (2012), then for the entire host we would obtain $S F R_{[\mathrm{CI}]} \sim 0.465 \pm 0.023 M_{\odot} \mathrm{yr}^{-1}$, which is also a factor of $\sim 1.7$ higher than the IR estimate. In contrast, the calibration of Herrera-Camus et al. (2015) gives a $S F R_{[\mathrm{C} \text { II }]} \sim 0.115 \pm 0.005 M_{\odot} \mathrm{yr}^{-1}$, that is lower than $\mathrm{SFR}_{\mathrm{IR}}$. It is unclear why this calibration is a factor of $\sim 6$ lower than that of De Looze et al. (2014). Yet, we use the calibration of
De Looze et al. (2014) given the larger sample size it is based on (530 versus 46 ), and because the [C II]/FIR luminosity ratio of the GRB 980425 host is indeed larger than that of other galaxies (Fig. 4), so it is expected that $S F R_{[\mathrm{C} \text { II }]}>S F R_{\mathrm{IR}}$.

The APEX $\mathrm{CO}(2-1)$ spectrum of the entire host is shown in Fig. 3 and the $\mathrm{CO}$ flux, luminosity, and the resulting molecular gas mass are presented in Table 2. The CO-derived molecular mass of $\sim 5.4 \times 10^{7} M_{\odot}$ is lower than the [C II]-derived molecular mass of $\sim 1.3 \times 10^{8} M_{\odot}$. Both estimates are based on a similar area over which the emission is summed (see Fig. 1), and are lower than the upper limit of $3 \times 10^{8} M_{\odot}$ derived by Hatsukade et al. (2007). Figure 7 demonstrates the high $\left[\mathrm{C}_{\text {II }}\right] / \mathrm{CO}(1-0)$ luminosity ratio of the GRB 980425 host 


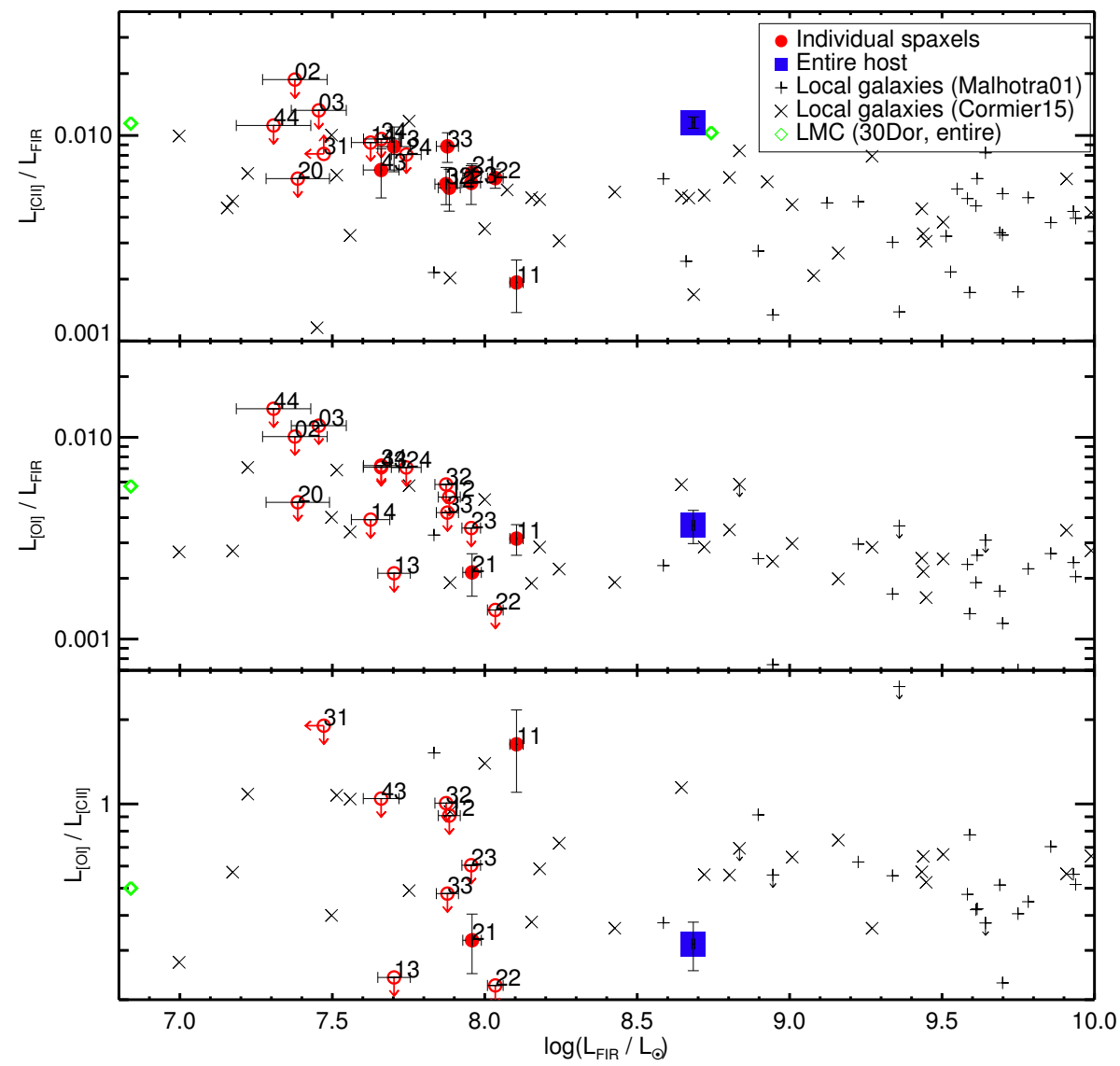

Fig. 4. [C II $]$-to-continuum (top), [O I $]$-tocontinuum (middle), and [O I]-to-[C II] (bottom) luminosity ratios as a function of FIR luminosity $(40-120 \mu \mathrm{m})$. Red circles denote PACS spaxels, blue square denotes the entire host, whereas plus signs, crosses and green diamonds represent normal local galaxies (Malhotra et al. 2001), dwarf galaxies (Cormier et al. 2015), and the Large Magellanic Cloud; the point at a lower luminosity is 30 Doradus and that at the higher luminosity is the entire galaxy (Poglitsch et al. 1995; Israel et al. 1996). The GRB 980425 host has elevated [C II]/FIR and [O I]/FIR ratios for its FIR luminosity.

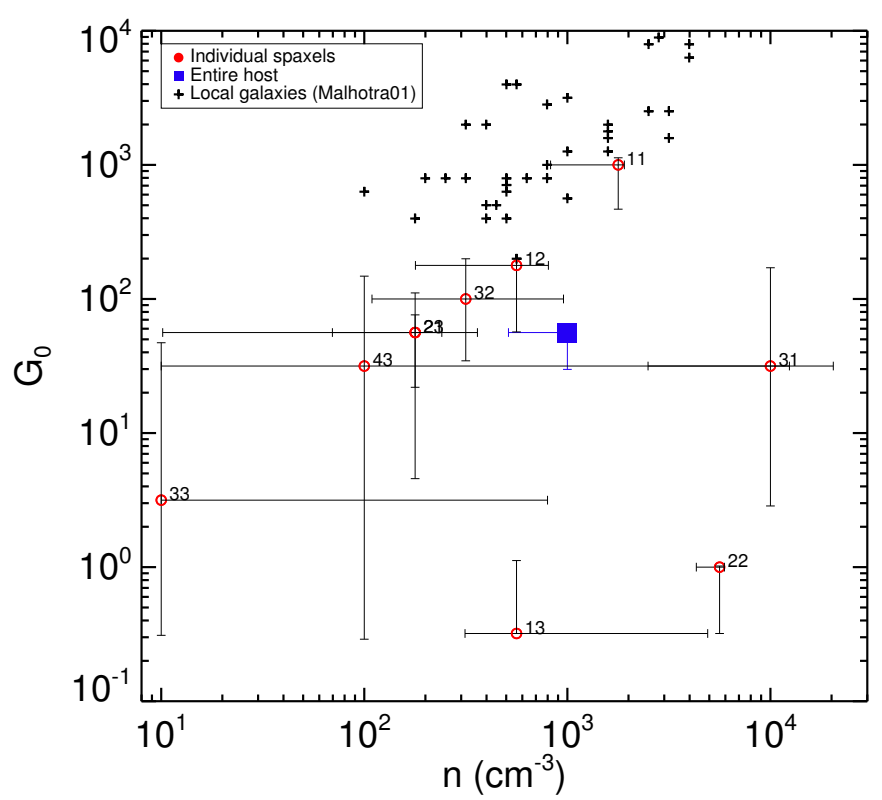

Fig. 5. Gas density vs. interstellar radiation field obtained from the PDR modelling. Red circles denote PACS spaxels, blue square denotes the entire host, whereas plus signs represent normal local galaxies (Malhotra et al. 2001). The WR region (spaxel 11) exhibits a high inferred radiation field and density.

compared with local galaxies (Crawford et al. 1985; Stacey et al. 1991).

The ALMA CO $(1-0)$ spectra extracted from the extents of PACS spaxels are shown in Fig. 9, whereas the spectrum of the WR region extracted within a $1.7^{\prime \prime}$ radius aperture is shown in
Fig. 10. No significant emission is detected, so we measured fluxes within $40 \mathrm{~km} \mathrm{~s}^{-1}$ width (the H I velocity width at a given position; Fig. 5 of Arabsalmani et al. 2015) at the frequency giving the highest conservative upper limit. These frequencies are shown as dotted lines in Figs. 9, and 10. Figure 3 shows the $\mathrm{CO}(1-0)$ spectrum of the entire host extracted within the region shown in Fig. 1 as a green circle. However, this region has a diameter of $\sim 27^{\prime \prime}$, whereas our ALMA observations are not sensitive to smooth emission extended over scales of more than $\sim 11^{\prime \prime}$. Hence, if such emission is present, then our $\mathrm{CO}(1-0)$ flux of the entire host is underestimated. The integrated flux ratio of $S_{\mathrm{CO}(2-1)} / S_{\mathrm{CO}(1-0)}>10(2 \sigma)$ is indeed higher than that of any other galaxy (Carilli \& Walter 2013), therefore our ALMA observations do not seem to probe the main emission component in the GRB 980425 host. The CO(1-0) fluxes, luminosities, and the resulting molecular gas masses are listed in Table 3. Again, Fig. 7 shows the high $[\mathrm{CII}] / \mathrm{CO}$ luminosity ratio of the spaxels that are detected at [C II].

The resulting star formation efficiency (SFE) is $L_{\mathrm{IR}} / L_{\mathrm{CO}(1-0)}^{\prime}=(95 \pm 19) L_{\odot} /\left(\mathrm{K} \mathrm{km} \mathrm{s}^{-1} \mathrm{pc}^{2}\right)$ (assuming $\left.L_{\mathrm{CO}(1-0)}^{\prime}=2 \times L_{\mathrm{CO}(2-1)}^{\prime}\right)$. This is higher than the average for local spirals of $\sim(48 \pm 7) L_{\odot} /\left(\mathrm{K} \mathrm{km} \mathrm{s}^{-1} \mathrm{pc}^{2}\right)$ derived by Daddi et al. (2010, their Fig. 13), close to the top end of the distribution. Together with three GRB hosts reported to be CO deficient (Hatsukade et al. 2014; Stanway et al. 2015b), the picture emerges that GRB hosts exhibit the lowest CO luminosities among star-forming galaxies, given their SFRs. On the other hand, if we assume that [C II] is a good tracer of molecular gas, then $\mathrm{SFE}=L_{\mathrm{IR}} / L_{[\mathrm{CII}]}^{\prime}=(40.4 \pm 2.0) L_{\odot} /\left(\mathrm{K} \mathrm{km} \mathrm{s}^{-1} \mathrm{pc}^{2}\right)$, in agreement with local galaxies.

In order to quantify further the potential molecular-gas deficiency of the GRB 980425 host, we used the relation between 
Table 1. Physical properties of each spaxel and the entire host.

\begin{tabular}{|c|c|c|c|c|c|c|}
\hline Reg & $\begin{array}{c}S F R_{\mathrm{IR}} \\
\left(M_{\odot} \mathrm{yr}^{-1}\right) \\
(2)\end{array}$ & $\begin{array}{c}S F R_{[\mathrm{CII}]} \\
\left(M_{\odot} \mathrm{yr}^{-1}\right) \\
(3)\end{array}$ & $\begin{array}{c}S F R_{[\mathrm{OI}]} \\
\left(M_{\odot} \mathrm{yr}^{-1}\right) \\
(4)\end{array}$ & $\begin{array}{c}n \\
\left(\mathrm{~cm}^{-3}\right) \\
(5)\end{array}$ & $\begin{array}{l}G_{0} \\
(6)\end{array}$ & $\begin{array}{c}M_{\mathrm{H}_{2},[\mathrm{CII}]} \\
\left(10^{6} M_{\odot}\right) \\
(7) \\
(7)\end{array}$ \\
\hline Host & $0.260 \pm 0.080$ & $0.668 \pm 0.040$ & $0.287 \pm 0.054$ & $1000_{-}^{+} 34$ & $56.2_{-}^{+}$ & $127.3 \pm 7.9$ \\
\hline 44 & $0.008 \pm 0.002$ & $0.012 \pm 0.007$ & $0.009 \pm 0.019$ & ${ }^{-}{ }^{487}$ & $\ldots$ & $2.3 \pm 1.4$ \\
\hline 43 & $0.017 \pm 0.002$ & $0.036 \pm 0.008$ & $0.015 \pm 0.019$ & $100_{-\quad 90}^{+12302}$ & $31.6_{-31.3}^{+116.3}$ & $7.1 \pm 1.6$ \\
\hline 42 & $0.006 \pm 0.002$ & $0.023 \pm 0.013$ & $-0.012 \pm 0.012$ & $\ldots$ & $\ldots$ & $4.5 \pm 2.5$ \\
\hline 41 & $-0.003 \pm 0.002$ & $-0.034 \pm 0.020$ & $-0.023 \pm 0.015$ & $\ldots$ & $\ldots$ & $-6.8 \pm 3.9$ \\
\hline 40 & $-0.000 \pm 0.002$ & $-0.021 \pm 0.013$ & $0.007 \pm 0.022$ & $\cdots$ & $\cdots$ & $-4.1 \pm 2.5$ \\
\hline 34 & $0.017 \pm 0.002$ & $0.029 \pm 0.011$ & $-0.015 \pm 0.035$ & $\ldots$ & $\ldots$ & $5.8 \pm 2.1$ \\
\hline 33 & $0.028 \pm 0.002$ & $0.078 \pm 0.011$ & $0.016 \pm 0.018$ & $10_{-}^{+} \quad 789$ & $3.2_{-}^{+} \quad 44.0$ & $15.2 \pm 2.1$ \\
\hline 32 & $0.028 \pm 0.002$ & $0.050 \pm 0.009$ & $0.038 \pm 0.016$ & $316_{-}^{+}$ & $\begin{array}{l}100.0_{-}^{+} 99.5 \\
5.4\end{array}$ & $9.9 \pm 1.8$ \\
\hline 31 & $0.006 \pm 0.002$ & $0.028 \pm 0.009$ & $0.044 \pm 0.015$ & $10000_{-7503}^{+10399}$ & $31.6_{-28.7}^{+139.4}$ & $5.5 \pm 1.8$ \\
\hline 30 & $0.003 \pm 0.002$ & $-0.009 \pm 0.011$ & $0.015 \pm 0.015$ & $\ldots$ & $\ldots$ & $-1.7 \pm 2.2$ \\
\hline 24 & $0.021 \pm 0.002$ & $0.030 \pm 0.011$ & $0.035 \pm 0.014$ & $\cdots$ & $\cdots$ & $5.9 \pm 2.1$ \\
\hline 23 & $0.034 \pm 0.002$ & $0.062 \pm 0.012$ & $0.030 \pm 0.011$ & 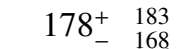 & $\begin{array}{l}56.2_{-51.6}^{+54.8} \\
0\end{array}$ & $12.1 \pm 2.4$ \\
\hline 22 & $0.040 \pm 0.002$ & $0.079 \pm 0.007$ & $0.008 \pm 0.008$ & $5620_{-13}^{+}$ & $\begin{array}{l}1.0_{-}^{+} \\
0.8 \\
0.7\end{array}$ & $15.3 \pm 1.4$ \\
\hline 21 & $0.034 \pm 0.002$ & $0.070 \pm 0.006$ & $0.032 \pm 0.007$ & $\begin{array}{r}178_{-}^{+} \quad 63 \\
\end{array}$ & $\begin{array}{l}56.2_{-34.3}^{+} 19.9 \\
0\end{array}$ & $13.6 \pm 1.1$ \\
\hline 20 & $0.009 \pm 0.002$ & $-0.004 \pm 0.010$ & $-0.000 \pm 0.010$ & $\ldots$ & $\ldots$ & $-0.7 \pm 2.1$ \\
\hline 14 & $0.016 \pm 0.002$ & $0.025 \pm 0.010$ & $-0.004 \pm 0.015$ & $\cdots$ & $\cdots$ & $5.0 \pm 2.0$ \\
\hline 13 & $0.019 \pm 0.002$ & $0.052 \pm 0.011$ & $-0.011 \pm 0.014$ & $562_{-}^{+} \quad 4347$ & $\begin{array}{ll}0.3_{-}^{+} & 0.8 \\
0.0\end{array}$ & $10.1 \pm 2.1$ \\
\hline 12 & $0.028 \pm 0.002$ & $0.050 \pm 0.010$ & $0.038 \pm 0.012$ & $562_{-}^{+} 245$ & $178.0_{-121.2}^{+21.9}$ & $9.7 \pm 2.1$ \\
\hline 11 & $0.047 \pm 0.002$ & $0.028 \pm 0.008$ & $0.065 \pm 0.011$ & $\begin{array}{l}1780_{-}^{+} \quad 129 \\
953\end{array}$ & $1000.0_{-532.6}^{+129.9}$ & $5.6 \pm 1.6$ \\
\hline 10 & $0.005 \pm 0.002$ & $-0.016 \pm 0.007$ & $0.009 \pm 0.018$ & ... & $\ldots$ & $-3.1 \pm 1.5$ \\
\hline 04 & $0.003 \pm 0.002$ & $0.020 \pm 0.014$ & $-0.022 \pm 0.018$ & $\cdots$ & $\cdots$ & $3.9 \pm 2.7$ \\
\hline 03 & $0.011 \pm 0.002$ & $0.025 \pm 0.009$ & $0.017 \pm 0.018$ & $\ldots$ & $\ldots$ & $5.0 \pm 1.8$ \\
\hline 02 & $0.009 \pm 0.002$ & $0.026 \pm 0.013$ & $0.007 \pm 0.016$ & $\cdots$ & $\cdots$ & $5.1 \pm 2.5$ \\
\hline 01 & $0.004 \pm 0.002$ & $0.012 \pm 0.007$ & $0.017 \pm 0.014$ & $\cdots$ & $\cdots$ & $2.4 \pm 1.4$ \\
\hline 00 & $0.000 \pm 0.002$ & $-0.016 \pm 0.013$ & $-0.052 \pm 0.016$ & $\cdots$ & $\cdots$ & $-3.2 \pm 2.6$ \\
\hline
\end{tabular}

Notes. (1) The entire host or the spaxel number. (2) Star formation rates (SFRs) from the total $(8-1000 \mu \mathrm{m})$ luminosity using $S F R / M_{\odot} \mathrm{yr}^{-1}=$ $10^{-10} L_{\mathrm{IR}} / L_{\odot}$ assuming the Chabrier (2003) initial mass function. (3), (4) SFRs from the [C II] and [O I] lines, respectively, using the calibration of De Looze et al. (2014). (5) Gas density. (6) Strength of the interstellar radiation field in Habing unit (solar neighbourhood value), both derived via the PDR modelling (Sect. 3). (7) Molecular gas mass estimated assuming $L_{\mathrm{CO}(1-0)}^{\prime}=L_{[\mathrm{C} \mathrm{II]}}^{\prime}$ (see Sect. 3) and the Galactic CO-to- $\mathrm{H}_{2}$ conversion factor $\alpha_{\mathrm{CO}}=5 M_{\odot} /\left(\mathrm{K} \mathrm{km} \mathrm{s}^{-1} \mathrm{pc}^{2}\right)$.

Table 2. APEX CO(2-1) line fluxes and luminosities.

\begin{tabular}{lcccccc}
\hline \hline Reg & $\begin{array}{c}F_{\text {int }} \\
\left(\mathrm{Jy} \mathrm{km} \mathrm{s}^{-1}\right)\end{array}$ & $S / N$ & $\begin{array}{c}F_{\mathrm{int}} \\
\left(10^{-17} \mathrm{~W} \mathrm{~m}^{-2}\right)\end{array}$ & $\begin{array}{c}L \\
\left(10^{5} L_{\odot}\right)\end{array}$ & $\begin{array}{c}L^{\prime} \\
\left(10^{6} \mathrm{~K} \mathrm{~km} \mathrm{~s}^{-1} \mathrm{pc}^{2}\right)\end{array}$ & $\begin{array}{c}M_{\mathrm{H}_{2}, \mathrm{CO}} \\
\left(10^{6} M_{\odot}\right)\end{array}$ \\
$(1)$ & $(2)$ & $(3)$ & $(4)$ & $(5)$ & $(6)$ & $(7)$ \\
\hline Host & $6.5 \pm 1.3$ & 5.1 & $0.0050 \pm 0.0010$ & $0.021 \pm 0.004$ & $5.4 \pm 1.1$ & $54.2 \pm 10.7$ \\
\hline
\end{tabular}

Notes. (1) The entire host. (2) Integrated flux within dotted lines in Fig. 3. (3) Signal-to-noise ratio of the line. (4) Integrated flux in W $\mathrm{m}^{-2}$. (5) Line luminosity in solar luminosity. (6) Line luminosity using Eq. (3) in Solomon et al. (1997). (7) Molecular gas mass estimated assuming $L_{\mathrm{CO}(1-0)}^{\prime}=2 \times L_{\mathrm{CO}(2-1)}^{\prime}\left(\right.$ see Sect. 3) and the Galactic CO-to- $\mathrm{H}_{2}$ conversion factor $\alpha_{\mathrm{CO}}=5 M_{\odot} /\left(\mathrm{K} \mathrm{km} \mathrm{s}^{-1} \mathrm{pc}^{2}\right)$.

the metallicity, atomic gas, and molecular gas for dwarf galaxies provided by Filho et al. (2016, their Sect. 4), based on the calibration of Amorín et al. (2016): $\log \left(M_{\mathrm{H}_{2}}\right)=1.2 \log \left(M_{\mathrm{HI}}\right)-$ $1.5 \times[12+\log (\mathrm{O} / \mathrm{H})-8.7]-2.2$. For its atomic gas mass $\log \left(M_{\mathrm{HI}} / M_{\odot}\right) \sim 8.849$ (Michałowski et al. 2015) and metallicity $12+\log (\mathrm{O} / \mathrm{H}) \sim 8.6$ (Sollerman et al. 2005), the GRB 980425 host should have $M_{\mathrm{H}_{2}} \sim 4 \times 10^{8} M_{\odot}, \sim 7$ times higher than the $\mathrm{CO}$ estimate (Table 2) and $\sim 3$ times higher than the [C II] estimate (Table 1). Hence, the GRB 980425 host has a low molecular gas mass for its atomic gas mass and metallicity. A similar conclusion can be obtained from the relation between SFR, CO luminosity, and metallicity presented in Hunt et al. (2015, their Fig. 5): $\log \left(S F R / L^{\prime}{ }_{\mathrm{CO}}\right)=-2.25 \times[12+\log (\mathrm{O} / \mathrm{H})]+11.31$.
According to this relation $S F R / L^{\prime}$ CO of the GRB 980425 host should be equal to $9.1 \times 10^{-9} M_{\odot} \mathrm{yr}^{-1} /\left(\mathrm{K} \mathrm{km} \mathrm{s}^{-1} \mathrm{pc}^{2}\right)$, whereas using SFR $_{\mathrm{IR}}$ the measured value is $\sim 2.5$ times higher: $24 \pm 9 \times$ $10^{-9} M_{\odot} \mathrm{yr}^{-1} /\left(\mathrm{K} \mathrm{km} \mathrm{s}^{-1} \mathrm{pc}^{2}\right)$, indicating low CO luminosity for its SFR and metallicity.

\section{Discussion}

\subsection{Recent inflow of atomic gas from the intergalactic medium triggering star formation}

Summarising, we have the following pieces of information about the GRB 980425 host: $i$ ) it has elevated [C II]/FIR and [O I]/FIR ratios (Fig. 4) and higher values of SFRs derived from line 


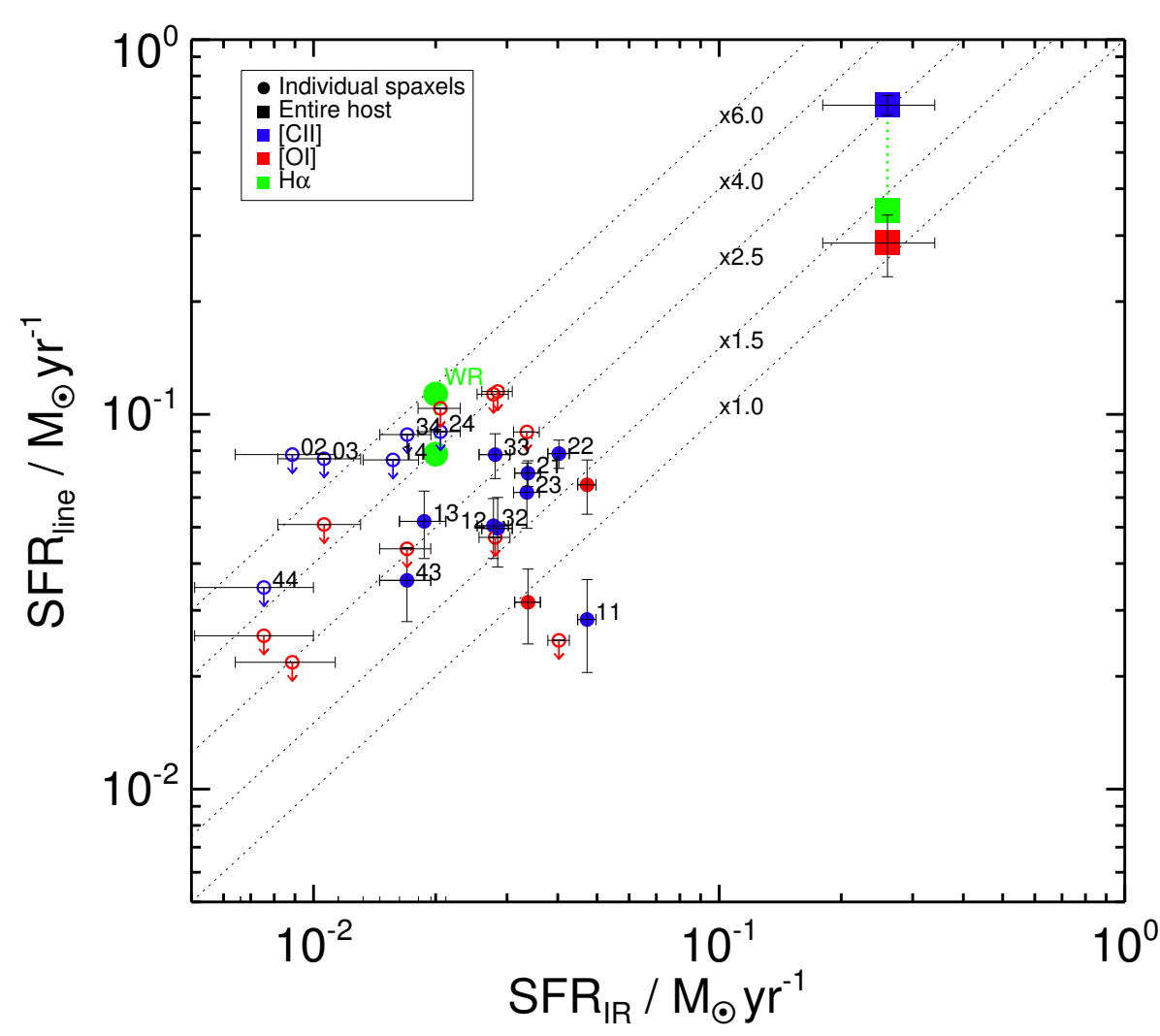

Fig. 6. SFRs derived from IR luminosity $(8-1000 \mu \mathrm{m}$; with the calibration of Kennicutt 1998) vs. those derived from [C II] (blue), [O I] (red; with the calibration of De Looze et al. 2014), and $\mathrm{H} \alpha$ (green, higher values include dust correction as derived by Christensen et al. 2008) lines. Circles denote PACS spaxels, and squares denote the entire host. The IR estimates are from Michałowski et al. (2014b). The $\mathrm{H} \alpha$ estimate for the WR region is from Christensen et al. (2008), and for the entire host from Sollerman et al. (2005). Dotted lines show the locations at which the $\mathrm{SFR}_{\text {line }}$ is higher than the $\mathrm{SFR}_{\mathrm{IR}}$ by the indicated factors. The SFRs derived from line ([C II], [O I $], \mathrm{H} \alpha)$ indicators are higher than those from continuum (UV, IR, radio) indicators.

Table 3. ALMA CO(1-0) line fluxes and luminosities of each spaxel and the WR region.

\begin{tabular}{|c|c|c|c|c|c|c|}
\hline $\operatorname{Reg}$ & $\begin{array}{c}F_{\text {int }} \\
\left(\mathrm{Jy} \mathrm{km} \mathrm{s}^{-1}\right) \\
(2)\end{array}$ & $S / N$ & $\begin{array}{c}F_{\mathrm{int}} \\
\left(10^{-22} \mathrm{~W} \mathrm{~m} \mathrm{~m}^{-2}\right) \\
(4)\end{array}$ & $\begin{array}{c} \\
\left(L_{\odot}\right) \\
(5)\end{array}$ & $\begin{array}{c}L^{\prime} \\
\left(10^{6} \mathrm{~K} \mathrm{~km} \mathrm{~s}^{-1} \mathrm{pc}^{2}\right) \\
(6)\end{array}$ & $\begin{array}{c}M_{\mathrm{H}_{2}, \mathrm{CO}} \\
\left(10^{6} M_{\odot}\right) \\
(7)\end{array}$ \\
\hline Host & $-0.406 \pm 0.316$ & -1.3 & $-15.6 \pm 12.1$ & $-66.4 \pm 51.7$ & $-1.36 \pm 1.05$ & $-6.8 \pm 5.3$ \\
\hline WR & $0.058 \pm 0.049$ & 1.2 & $2.2 \pm 1.9$ & $9.5 \pm 8.1$ & $0.19 \pm 0.16$ & $1.0 \pm 0.8$ \\
\hline 44 & $0.271 \pm 0.125$ & 2.2 & $10.4 \pm 4.8$ & $44.4 \pm 20.4$ & $0.91 \pm 0.42$ & $4.5 \pm 2.1$ \\
\hline 43 & $0.318 \pm 0.141$ & 2.3 & $12.2 \pm 5.4$ & $52.1 \pm 23.0$ & $1.06 \pm 0.47$ & $5.3 \pm 2.3$ \\
\hline 42 & $0.209 \pm 0.109$ & 1.9 & $8.1 \pm 4.2$ & $34.3 \pm 17.8$ & $0.70 \pm 0.36$ & $3.5 \pm 1.8$ \\
\hline 41 & $-0.040 \pm 0.132$ & -0.3 & $-1.5 \pm 5.1$ & $-6.5 \pm 21.6$ & $-0.13 \pm 0.44$ & $-0.7 \pm 2.2$ \\
\hline 40 & $0.099 \pm 0.128$ & 0.8 & $3.8 \pm 4.9$ & $16.3 \pm 21.0$ & $0.33 \pm 0.43$ & $1.7 \pm 2.1$ \\
\hline 34 & $0.301 \pm 0.132$ & 2.3 & $11.6 \pm 5.1$ & $49.2 \pm 21.6$ & $1.00 \pm 0.44$ & $5.0 \pm 2.2$ \\
\hline 33 & $0.003 \pm 0.126$ & 0.0 & $0.1 \pm 4.9$ & $0.5 \pm 20.7$ & $0.01 \pm 0.42$ & $0.1 \pm 2.1$ \\
\hline 32 & $0.118 \pm 0.124$ & 0.9 & $4.5 \pm 4.8$ & $19.3 \pm 20.4$ & $0.39 \pm 0.42$ & $2.0 \pm 2.1$ \\
\hline 31 & $0.288 \pm 0.140$ & 2.1 & $11.1 \pm 5.4$ & $47.1 \pm 22.8$ & $0.96 \pm 0.47$ & $4.8 \pm 2.3$ \\
\hline 30 & $0.263 \pm 0.111$ & 2.4 & $10.1 \pm 4.3$ & $43.1 \pm 18.2$ & $0.88 \pm 0.37$ & $4.4 \pm 1.9$ \\
\hline 24 & $0.154 \pm 0.103$ & 1.5 & $5.9 \pm 4.0$ & $25.1 \pm 16.9$ & $0.51 \pm 0.34$ & $2.6 \pm 1.7$ \\
\hline 23 & $0.267 \pm 0.137$ & 2.0 & $10.3 \pm 5.3$ & $43.7 \pm 22.4$ & $0.89 \pm 0.46$ & $4.5 \pm 2.3$ \\
\hline 22 & $0.178 \pm 0.108$ & 1.6 & $6.8 \pm 4.2$ & $29.1 \pm 17.7$ & $0.59 \pm 0.36$ & $3.0 \pm 1.8$ \\
\hline 21 & $0.170 \pm 0.119$ & 1.4 & $6.6 \pm 4.6$ & $27.9 \pm 19.5$ & $0.57 \pm 0.40$ & $2.8 \pm 2.0$ \\
\hline 20 & $0.225 \pm 0.121$ & 1.9 & $8.7 \pm 4.7$ & $36.9 \pm 19.9$ & $0.75 \pm 0.41$ & $3.8 \pm 2.0$ \\
\hline 14 & $0.009 \pm 0.114$ & 0.1 & $0.4 \pm 4.4$ & $1.5 \pm 18.7$ & $0.03 \pm 0.38$ & $0.2 \pm 1.9$ \\
\hline 13 & $0.102 \pm 0.129$ & 0.8 & $3.9 \pm 5.0$ & $16.7 \pm 21.1$ & $0.34 \pm 0.43$ & $1.7 \pm 2.2$ \\
\hline 12 & $0.156 \pm 0.109$ & 1.4 & $6.0 \pm 4.2$ & $25.5 \pm 17.8$ & $0.52 \pm 0.36$ & $2.6 \pm 1.8$ \\
\hline 11 & $0.208 \pm 0.111$ & 1.9 & $8.0 \pm 4.3$ & $34.1 \pm 18.1$ & $0.70 \pm 0.37$ & $3.5 \pm 1.9$ \\
\hline 10 & $0.116 \pm 0.114$ & 1.0 & $4.4 \pm 4.4$ & $18.9 \pm 18.6$ & $0.39 \pm 0.38$ & $1.9 \pm 1.9$ \\
\hline 04 & $0.114 \pm 0.117$ & 1.0 & $4.4 \pm 4.5$ & $18.6 \pm 19.2$ & $0.38 \pm 0.39$ & $1.9 \pm 2.0$ \\
\hline 03 & $0.124 \pm 0.123$ & 1.0 & $4.8 \pm 4.7$ & $20.3 \pm 20.1$ & $0.41 \pm 0.41$ & $2.1 \pm 2.1$ \\
\hline 02 & $0.130 \pm 0.116$ & 1.1 & $5.0 \pm 4.5$ & $21.3 \pm 19.0$ & $0.43 \pm 0.39$ & $2.2 \pm 1.9$ \\
\hline 01 & $0.248 \pm 0.121$ & 2.0 & $9.5 \pm 4.7$ & $40.5 \pm 19.9$ & $0.83 \pm 0.41$ & $4.1 \pm 2.0$ \\
\hline 00 & $0.160 \pm 0.115$ & 1.4 & $6.2 \pm 4.4$ & $26.2 \pm 18.9$ & $0.53 \pm 0.39$ & $2.7 \pm 1.9$ \\
\hline
\end{tabular}

Notes. (1) The entire host, the WR region, or the spaxel number. (2) Integrated flux within dotted lines in Figs. 9 and 10. (3) Signal-to-noise ratio of the line. (4) Integrated flux in $\mathrm{W} \mathrm{m}^{-2}$. (5) Line luminosity in solar luminosity. (6) Line luminosity using Eq. (3) in Solomon et al. (1997). (7) Molecular gas mass estimated assuming the Galactic CO-to- $\mathrm{H}_{2}$ conversion factor $\alpha_{\mathrm{CO}}=5 M_{\odot} /\left(\mathrm{K} \mathrm{km} \mathrm{s}^{-1} \mathrm{pc}^{2}\right)$. 


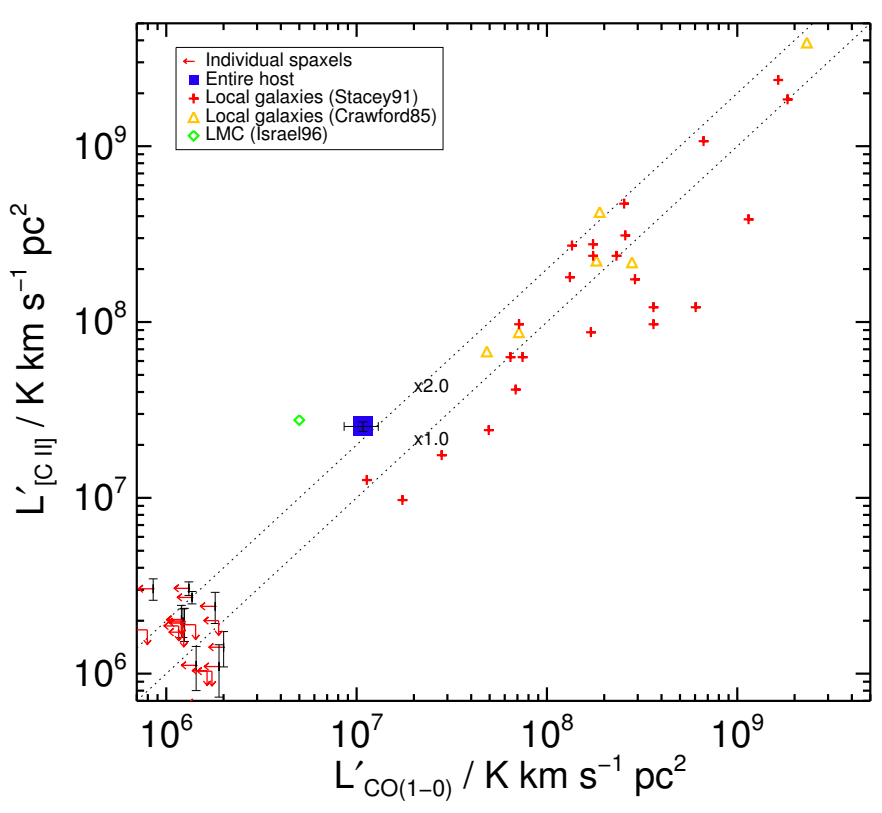

Fig. 7. [C II] luminosity as a function $\mathrm{CO}(1-0)$ luminosity of individual spaxels (red arrows), the entire GRB 980425 host (blue square), local galaxies (plus signs, diamonds, and crosses; Stacey et al. 1991; Crawford et al. 1985) and the LMC (green diamond; Israel et al. 1996). The GRB 980425 host has an elevated [C II]/CO ratio compared with local galaxies.

([C II], [O I] , H $\alpha$ ) than from continuum (UV, IR, radio) indicators (Fig. 6); ii) its [C II] emission exhibits a normal radial profile, whereas [OI] emission is concentrated close to the WR region (Fig. 2), leading to high inferred radiation field and density (Fig. 5); and iii) its CO luminosity is at the lower end of the distribution for other galaxies, leading to an elevated [C II]/CO ratio (Fig. 7) and $M_{\mathrm{H}_{2}}$ derived from CO lower by a factor of $\sim 2$ than that inferred from [C II].

All these observables can be explained by the hypothesis presented in Michałowski et al. (2015) that this galaxy in particular, and possibly most GRB hosts, experienced a very recent inflow of atomic gas triggering star formation. Indeed, $\mathrm{HI}$ is concentrated close to the position of the WR region (our spaxels 11, 12, 21; Arabsalmani et al. 2015). In this scenario the newly acquired atomic gas quickly becomes cool and dense, leading to intense star formation (giving rise to the birth of the GRB 980425 progenitor), and explaining the high [O I] and high derived radiation field and density. The accretion event disturbs the gas reservoir in other parts of the galaxy as well, and therefore the total SFR and ionised carbon emission are enhanced. However, dust has not had time to build up and the reprocessing of stellar emission by dust is much slower (see below), and so the infrared emission is still low giving rise to high [C II]/FIR and $S F R_{[\mathrm{C} \mathrm{II}]} / S F R_{\mathrm{IR}}$ ratios.

We note that high gas density close to the GRB position (Fig. 5) is consistent with the conclusion of Michałowski et al. (2014b) based on the properties of the WR region. Far-infrared spectroscopy of other GRB hosts is needed to test whether having a high density and radiation field is a condition necessary for GRB explosions.

Our $[\mathrm{CII}]$ and $[\mathrm{OI}]$ maps are not of enough resolution to investigate the spatial distribution of the resulting specific SFR $\left(s S F R \equiv S F R / M_{*}\right)$. However, stellar mass is distributed relatively smoothly across the host, and in particular the WR region does not have an exceptionally high or low mass
(Christensen et al. 2008; Michałowski et al. 2009). Hence, SFR and sSFR distributions based on $\mathrm{H} \alpha$ emission are very similar (Christensen et al. 2008).

Our data allows us to estimate the timescale of the required gas inflow. The line emission ([C II], [O I], $\mathrm{H} \alpha$ ) is connected with the most massive (O type) stars, hence it traces very recent star formation that happened during the last $\sim 10 \mathrm{Myr}$. On the other hand, UV, IR, and radio emission traces the average SFR over the last $100 \mathrm{Myr}$ (Kennicutt 1998), so the continuum emission is relatively insensitive to the enhancement in star formation due to very recent gas inflow. Hence, our hypothesis requires the inflow to happen at most a few tens of Myr ago, so that the line SFR indicators still give higher values than the continuum SFR indicators.

The timescale of less than a few tens of Myr is consistent with the estimates of the age of the GRB progenitor for GRB 980425 in particular, and for all GRBs in general. Sollerman et al. (2005) obtained the stellar age of the region in which GRB 980425 exploded of $\sim 6 \mathrm{Myr}$, which is consistent with the timescale we propose for the trigger of star formation. Similarly, if the progenitor of GRB 980425 was a runaway star expelled from the WR region (Hammer et al. 2006; van den Heuvel \& Portegies Zwart 2013), then it would need only $~ 3-6$ Myr to reach its explosion site assuming a reasonable kick velocity of $\sim 130-260 \mathrm{~km} \mathrm{~s}^{-1}$. Similar ages of stellar progenitors were derived for other GRBs (Thöne et al. 2008; Östlin et al. 2008).

On the other hand, the free-fall time is

$t_{\mathrm{ff}}=\left(\frac{3 \pi}{32 G \rho}\right)^{1 / 2} \approx 1.6 \mathrm{Myr}\left(\frac{n}{1000 \mathrm{~cm}^{-3}}\right)^{-1 / 2}$,

where $G$ is the gravitational constant, $\rho$ is gas density, and $n$ is the number density for which we assumed $\rho=n m_{\mathrm{p}}$, where $m_{\mathrm{p}}$ is the proton mass (i.e. assuming atomic gas only). For our measured density of $n \sim 1000 \mathrm{~cm}^{-3}$ (Fig. 5 and Table 1) the free-fall time is a few Myr. Star formation starts after one to a few $t_{\mathrm{ff}}$, so if, as proposed above, the inflow happened a few tens of Myr ago, then this is long enough for star formation to start.

We investigate the relation between SFRs of other GRB hosts derived from line and continuum emission in Fig. 8, in which we compiled the $\mathrm{H} \alpha$, [O II $] \lambda 3727$, UV, IR and radio estimates (Bloom et al. 1998, 2001; Djorgovski et al. 2001, 2003; Price et al. 2002; Garnavich et al. 2003; Christensen et al. 2004; Prochaska et al. 2004; Gorosabel et al. 2005; Sollerman et al. 2005, 2006; Castro Cerón et al. 2006, 2010; Della Valle et al. 2006; Thöne et al. 2008; Michałowski et al. 2009, 2012b, 2014b, 2015; Savaglio et al. 2009; Levesque et al. 2010b,a; Stanway et al. 2010; Watson et al. 2011; Hjorth et al. 2012; Jakobsson et al. 2012; Perley \& Perley 2013; Perley et al. 2015; Hunt et al. 2014a; Schady et al. 2014). If needed, we converted them to the Chabrier (2003) IMF by dividing the estimates based on the Salpeter (1955) IMF by 1.8. We note that the [O II] $\lambda 3727$ estimator is strongly metallicity-dependent (Kewley et al. 2004), but in our sample these estimates are consistent with those from $\mathrm{H} \alpha$ (mean ratio of $1.1 \pm 0.32$ ).

In principle it would also be advantageous to investigate sSFRs of GRB hosts. This would not change line-to-continuum ratios, but, if carried out properly, it could decrease the scatter in Fig. 8. This is because stellar mass estimates depend on many assumptions such as star formation histories, initial mass function, and stellar models (Michałowski et al. 2012a, 2014a; Pacifici et al. 2012; Mitchell et al. 2013; Simha et al. 2014), which may not be universal in the sample of GRB hosts. 


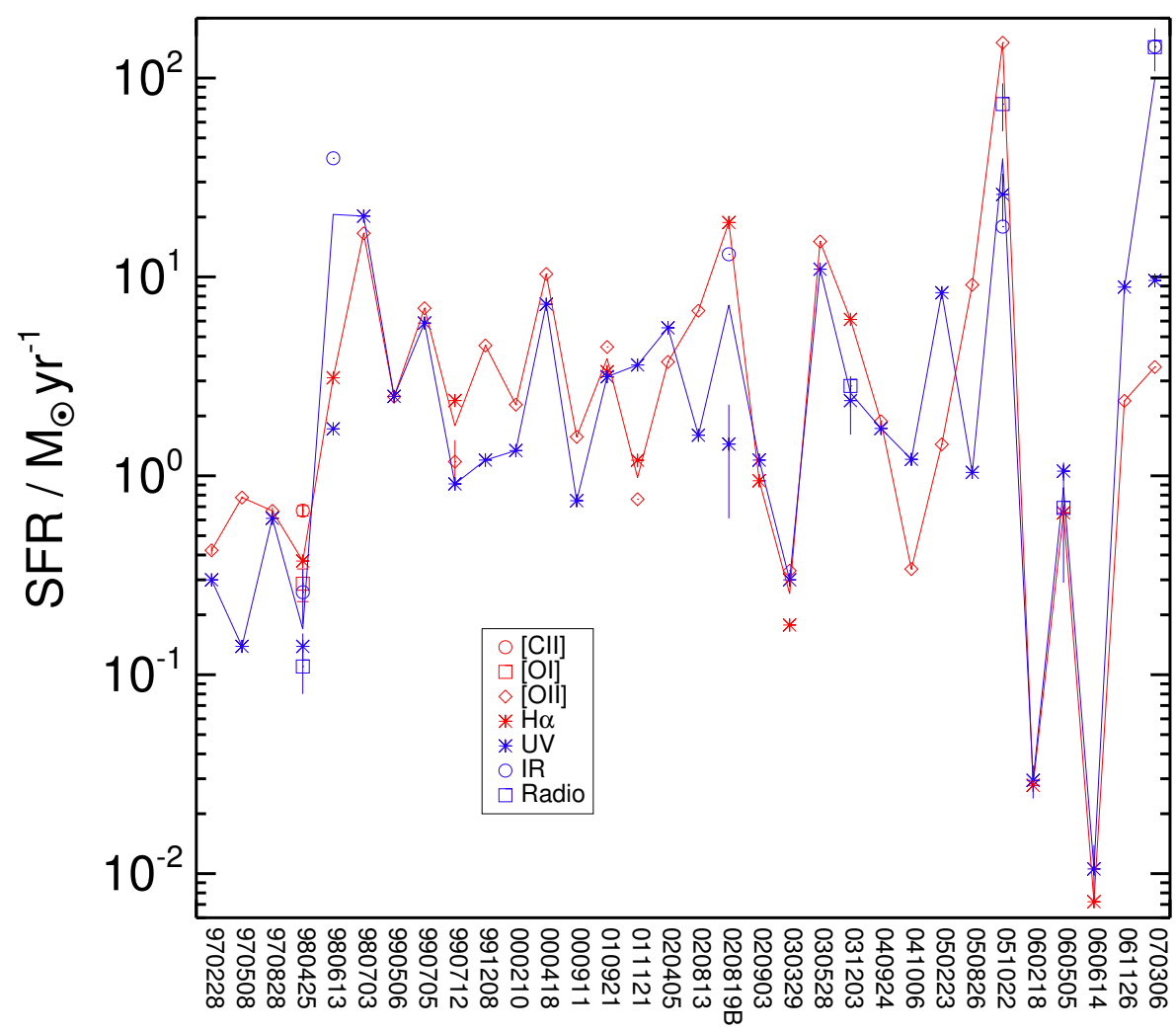

Fig. 8. Compilation of SFRs measured from emission lines (red; circles: [C II], squares: [O I], diamonds: [O II], asterisks: $\mathrm{H} \alpha$ ) and continuum indicators (blue; asterisks: ultraviolet, circles: infrared, squares: radio). The $x$-axis position corresponds to different GRB hosts. The red and blue lines show the average line- and continuum-based SFR for a given GRB host, respectively. The line-based indicators give on average systematically higher estimates.
However, the inhomogeneity of optical and near-infrared data for this sample renders this investigation beyond the scope of this paper.

In 20 out of 32 cases the line SFR is higher than the continuum SFR. The mean ratio of the SFR derived from line indicators to that from the continuum indicator is $1.74 \pm 0.32$. This is consistent with a very recent enhancement of star formation (reflected in stronger line emission, but not influencing the continuum yet) being common among GRB hosts. Hence, galaxies that have recently experienced inflow of gas may preferentially host stars exploding as GRBs. This may be due to the GRB metallicity bias favouring metalpoor environments (Yoon \& Langer 2005; Yoon et al. 2006; Woosley \& Heger 2006; Piranomonte et al. 2015; Schulze et al. 2015; Trenti et al. 2015; Vergani et al. 2015; Japelj et al. 2016; Perley et al. 2016). Hence the accretion of the metal-poor gas from the intergalactic medium is likely required to produce regions with low enough metallicity. Therefore GRBs may be used to select unique samples of galaxies suitable for the investigation of recent gas accretion.

The line and continuum indicators were calibrated so that they are consistent with each other on average for star-forming galaxies (see e.g. Wijesinghe et al. 2011; Davies et al. 2016; Wang et al. 2016), and therefore the higher line-based SFRs of GRB hosts are not due to systematic effects. Our interpretation of strong line emission is consistent with the investigation of other galaxies. The influence of the star formation history on the measured SFRs with various indicators was investigated by Guo et al. (2016), who interpreted low far-UV-to-H $\alpha$ SFR ratio of star-forming galaxies as a sign of recent starburst because this ratio anti-correlates with specific SFR measured with $\mathrm{H} \alpha$ (their Fig. 6, see also Sullivan et al. 2000; Iglesias-Páramo et al. 2004; Boselli et al. 2009; Lee et al. 2009; Meurer et al. 2009; Fumagalli et al. 2011; Weisz et al. 2012; da Silva et al. 2014).
Michałowski et al. (2015) proposed that star formation may proceed directly in the accreted atomic gas before the conversion to molecular gas. This is possible because the cooling timescale is much shorter than the $\mathrm{H}$ I-to- $\mathrm{H}_{2}$ conversion timescale, so just after the atomic gas is accreted it can start forming stars before it converts to the molecular phase (Glover \& Clark 2012; Krumholz 2012; Hu et al. 2016). As a result, molecular gas mass of GRB hosts is lower than what would be expected from their SFRs, which is partially fuelled by atomic gas.

\subsection{Alternative explanations}

Here we provide arguments that show why alternative explanations for our data are less likely.

High SFR/CO, [C II $] / \mathrm{CO}$, and $\mathrm{HI} / \mathrm{CO}$ ratios are usually found at low metallicities $(1 / 6-1 / 5$ solar or below; Poglitsch et al. 1995; Israel et al. 1996; Madden et al. 1997; Madden 2000; Rubin et al. 2009; Cormier et al. 2010; Hunt et al. 2014b, 2015; Amorín et al. 2016), so the GRB 980425 host in principle might be a normal metalpoor dwarf. However, the average metallicity of the host is $12+\log (\mathrm{O} / \mathrm{H}) \sim 8.6$ or $\sim 0.8$ solar (Sollerman et al. 2005), so it is difficult to advocate that its properties are due to metallicity effects. Indeed, as shown in the previous section, the GRB 980425 host has a low molecular gas mass even taking its metallicity into account. Moreover, the [C II]/FIR and [O I]/FIR ratios of the GRB 980425 host are higher than those of other local dwarf galaxies with similar FIR luminosity (Fig. 4).

Another possibility is that the GRB 980425 host is at the end of a star formation episode, which would imply molecular gas dissipation (hence weak CO emission) by massive stars (Hatsukade et al. 2014; Stanway et al. 2015b). However, in that case the WR region, which is forming stars most intensely, would be the most metal rich and dust free because dust would 


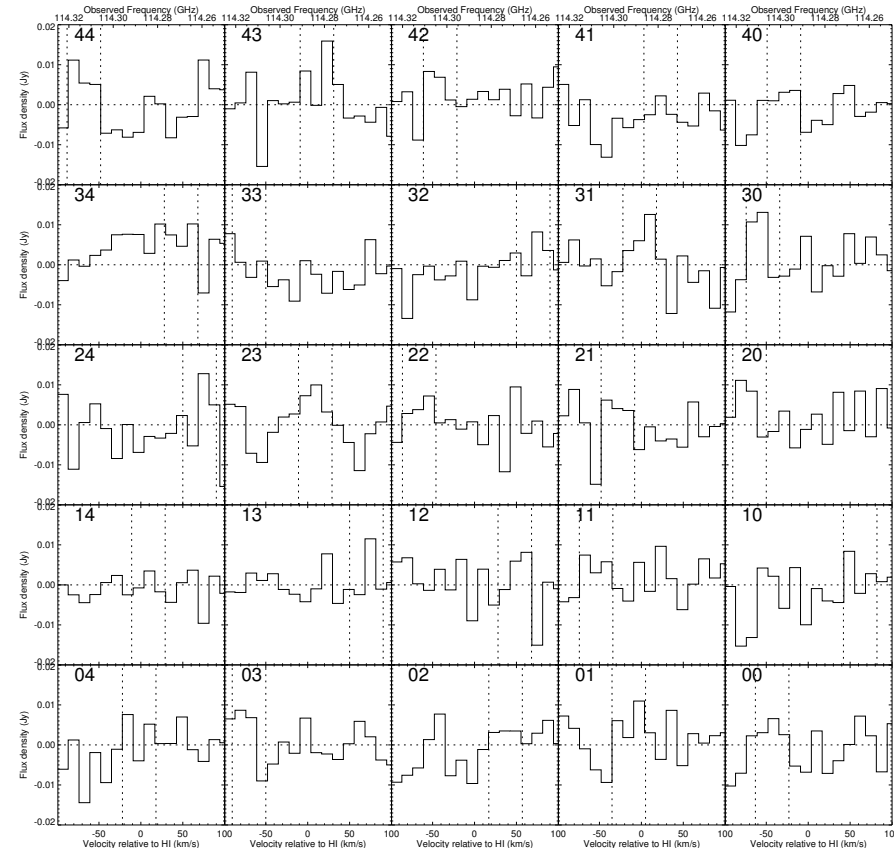

Fig. 9. ALMA $\mathrm{CO}(1-0)$ spectra of all spaxels. The integrated fluxes were measured within inner dotted lines. They are different for different spaxels because for each one we conservatively selected a $40 \mathrm{~km} \mathrm{~s}^{-1}$ region that gives the highest flux upper limit.

be destroyed together with molecular gas. On the contrary, the WR region is metal poor (Christensen et al. 2008) and dusty (Michałowski et al. 2014b). Moreover, if star formation activity was decreasing, then the [C II], [O I], and $\mathrm{H} \alpha$ emission would decrease almost instantaneously, whereas infrared emission would need a longer time to react. This would result in a lower (or at most equal) $\mathrm{SFR}_{\text {line }}$ than $\mathrm{SFR}_{\mathrm{IR}}$, contrary to observations (Fig. 6). The dust heating from older low-mass stars would make this effect even stronger.

In order to explain the $\mathrm{H}$ I concentration close to the WR region and the disturbed H I velocity field in the GRB 980425 host, Arabsalmani et al. (2015) invoked a minor merger scenario. This is actually not much different than our atomic gas inflow scenario because an $\mathrm{HI}$-dominated dwarf galaxy merging with the host is conceptually close to what we call an infalling H I cloud. However, we do not see the enhancement of [C II] close to the position of the WR region (similar to the H I concentration), so the smaller galaxy needs to be relatively unevolved in the merger scenario, such that it does not bring significant amount of carbon.

\section{Conclusions}

Using [CII], [OI] and CO spectroscopy we found that the GRB 980425 host has elevated [C II]/FIR and [O I]/FIR ratios and higher values of SFRs derived from line ([C II], [O I $], \mathrm{H} \alpha$ ) than from continuum (UV, IR, radio) indicators. [C II] emission exhibits a normal morphology, peaking at the galaxy centre, whereas $[\mathrm{OI}]$ is concentrated close to the GRB position and the nearby Wolf-Rayet region. The high [O I] flux indicates that there is high radiation field and high gas density at these positions, as derived from modelling of photo-dissociation regions. The [C II]/CO luminosity ratio of the GRB 980425 host is close to the highest values found for local star-forming galaxies. Indeed, its CO-derived molecular gas mass is low given its SFR

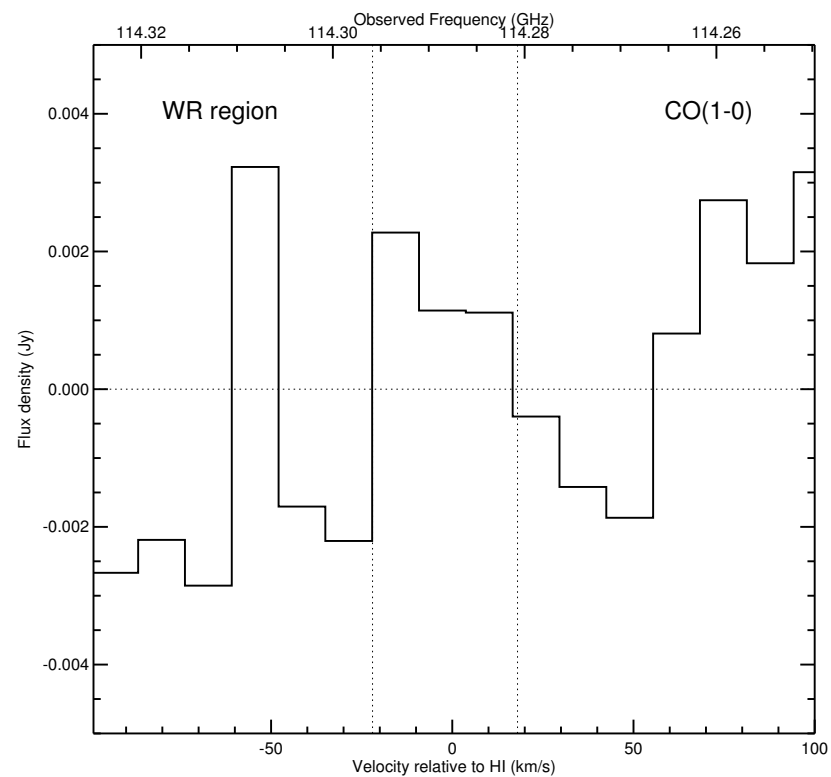

Fig. 10. ALMA $\mathrm{CO}(1-0)$ spectrum of the WR region within an $1.7^{\prime \prime}$ aperture. The flux was measured within inner dotted lines.

and metallicity, but the [C II]-derived molecular gas mass is close to the expected value.

The [O I] and $\mathrm{HI}$ concentrations and the high radiation field and density close to the GRB position are consistent with the hypothesis of a very recent (at most a few tens of Myr ago) inflow of atomic gas triggering star formation. In this scenario dust has not had time to build up, which explains high line-tocontinuum ratios. Such a recent enhancement of star formation activity would indeed manifest itself in high $S F R_{\text {line }} / S F R_{\text {continuum }}$ ratios because the line indicators are sensitive only to recent $(<10 \mathrm{Myr})$ activity, whereas the continuum indicators measure the SFR averaged over much longer periods ( 100 Myr). We found similarly high SFR ratios for other GRB hosts. This is consistent with a very recent enhancement of star formation that is common among GRB hosts, so galaxies that have recently experienced inflow of gas may preferentially host stars exploding as GRBs. Therefore GRBs may be used to select a unique sample of galaxies that is suitable for the investigation of recent gas accretion.

Acknowledgements. We thank Joanna Baradziej for help with improving this paper; Per Bergman, Carlos De Breuck, and Katharina Immer for help with the APEX observations; Tom Muxlow, and Eelco van Kampen with the ALMA observations; and our referee for useful comments. M.J.M. acknowledges the support of the UK Science and Technology Facilities Council. The Dark Cosmology Centre is funded by the Danish National Research Foundation. J.L.W. is supported by a European Union COFUND/Durham Junior Research Fellowship under EU grant agreement number 267209, and acknowledges additional support from STFC (ST/L00075X/1). A.K. acknowledges support from the Foundation for Polish Science (FNP) and the Polish National Science Center grant 2013/11/N/ST9/00400. A.J.C.T. acknowledges support from the Spanish Ministry Project AYA2015-71718-R. D.X. acknowledges the support by the One-Hundred-Talent Program of the Chinese Academy of Sciences, and by the Strategic Priority Research Program "Multi-wavelength Gravitational Wave Universe" of the Chinese Academy of Sciences (No. XDB23000000). PACS has been developed by a consortium of institutes led by MPE (Germany) and including UVIE (Austria); KU Leuven, CSL, IMEC (Belgium); CEA, LAM (France); MPIA (Germany); INAF-IFSI/OAA/OAP/OAT, LENS, SISSA (Italy); IAC (Spain). This development has been supported by the funding agencies BMVIT (Austria), ESA-PRODEX (Belgium), CEA/CNES (France), DLR (Germany), ASI/INAF (Italy), and CICYT/MCYT (Spain). This publication is based on data acquired with the Atacama Pathfinder Experiment (APEX). APEX is a collaboration between the Max-Planck-Institut fur Radioastronomie, the European Southern Observatory, and the Onsala Space 
Observatory. Based on observations collected at the European Organisation for Astronomical Research in the Southern Hemisphere under ESO programmes 096.D-0280 and 096.F-9302. This paper makes use of the following ALMA data: ADS/JAO.ALMA\#2011.0.00046.S. ALMA is a partnership of ESO (representing its member states), NSF (USA) and NINS (Japan), together with NRC (Canada) and NSC and ASIAA (Taiwan), in cooperation with the Republic of Chile. The Joint ALMA Observatory is operated by ESO, AUI/NRAO and NAOJ. This research has made use of the GHostS database (http://www. grbhosts.org), which is partly funded by Spitzer/NASA grant RSA Agreement No. 1287913; the NASA/IPAC Extragalactic Database (NED), which is operated by the Jet Propulsion Laboratory, California Institute of Technology, under contract with the National Aeronautics and Space Administration; SAOImage DS9, developed by Smithsonian Astrophysical Observatory (Joye \& Mandel 2003); and the NASA's Astrophysics Data System Bibliographic Services.

\section{References}

Amorín, R., Muñoz-Tuñón, C., Aguerri, J. A. L., \& Planesas, P. 2016, A\&A, 588, A23

Arabsalmani, M., Roychowdhury, S., Zwaan, M. A., Kanekar, N., \& Michałowski, M. J. 2015, MNRAS, 454, L51

Asplund, M., Grevesse, N., Sauval, A. J., Allende Prieto, C., \& Kiselman, D., 2004, A\&A, 417, 751

Bigiel, F., Leroy, A., Walter, F., et al. 2008, AJ, 136, 2846

Bigiel, F., Leroy, A., Walter, F., et al. 2010, AJ, 140, 1194

Bloom, J. S., Djorgovski, S. G., Kulkarni, S. R., \& Frail, D. A. 1998, ApJ, 507, L25

Bloom, J. S., Djorgovski, S. G., \& Kulkarni, S. R. 2001, ApJ, 554, 678 Boissier, S., Salvaterra, R., Le Floc'h, E., et al. 2013, A\&A, 557, A34

Bolatto, A. D., Wolfire, M., \& Leroy, A. K. 2013, ARA\&A, 51, 207

Boselli, A., Boissier, S., Cortese, L., et al. 2009, ApJ, 706, 1527

Carilli, C. L., \& Walter, F. 2013, ARA\&A, 51, 105

Castro Cerón, J. M., Michałowski, M. J., Hjorth, J., et al. 2006, ApJ, 653, L85

Castro Cerón, J. M., Michałowski, M. J., Hjorth, J., et al. 2010, ApJ, 721, 1919

Castro-Tirado, A. J., Bremer, M., McBreen, S., et al. 2007, A\&A, 475, 101

Chabrier, G. 2003, ApJ, 586, L133

Christensen, L., Hjorth, J., \& Gorosabel, J. 2004, A\&A, 425, 913

Christensen, L., Vreeswijk, P. M., Sollerman, J., et al. 2008, A\&A, 490, 45

Cormier, D., Madden, S. C., Hony, S., et al. 2010, A\&A, 518, L57

Cormier, D., Madden, S. C., Lebouteiller, V., et al. 2015, A\&A, 578, A53

Crawford, M. K., Genzel, R., Townes, C. H., \& Watson, D. M. 1985, ApJ, 291, 755

da Silva, R. L., Fumagalli, M., \& Krumholz, M. R. 2014, MNRAS, 444, 3275

Daddi, E., Bournaud, F., Walter, F., et al. 2010, ApJ, 713, 686

Davies, L. J. M., Driver, S. P., Robotham, A. S. G., et al. 2016, MNRAS, 461, 458

De Looze, I., Cormier, D., Lebouteiller, V., et al. 2014, A\&A, 568, A62

D'Elia, V., Fynbo, J. P. U., Covino, S., et al. 2010, A\&A, 523, A36

D'Elia, V., Fynbo, J. P. U., Goldoni, P., et al. 2014, A\&A, 564, A38

Della Valle, M., Chincarini, G., Panagia, N., et al. 2006, Nature, 444, 1050

Detmers, R. G., Langer, N., Podsiadlowski, P., \& Izzard, R. G. 2008, A\&A, 484, 831

Djorgovski, S. G., Frail, D. A., Kulkarni, S. R., et al. 2001, ApJ, 562, 654

Djorgovski, S. G., Bloom, J. S., \& Kulkarni, S. R. 2003, ApJ, 591, L13

Draine, B. T. 2009, ASP Conf. Ser., 414, 453

Elliott, J., Krühler, T., Greiner, J., et al. 2013, A\&A, 556, A23

Elmegreen, B. G., Kaufman, M., Bournaud, F., et al. 2016, ApJ, 823, 26

Filho, M. E., Sánchez Almeida, J., Amorín, R., et al. 2016, ApJ, 820, 109

Friis, M., De Cia, A., Krühler, T., et al. 2015, MNRAS, 451, 4686

Fruchter, A. S., Levan, A. J., Strolger, L., et al. 2006, Nature, 441, 463

Fumagalli, M., \& Gavazzi, G. 2008, A\&A, 490, 571

Fumagalli, M., da Silva, R. L., \& Krumholz, M. R. 2011, ApJ, 741, L26

Fynbo, J. P. U., Starling, R. L. C., Ledoux, C., et al. 2006, A\&A, 451, L47

Galama, T. J., Vreeswijk, P. M., van Paradijs, J., et al. 1998, Nature, 395, 670

Garnavich, P. M., Stanek, K. Z., Wyrzykowski, L., et al. 2003, ApJ, 582, 924

Georgy, C., Ekström, S., Meynet, G., et al. 2012, A\&A, 542, A29

Glover, S. C. O., \& Clark, P. C. 2012, MNRAS, 421, 9

Gorosabel, J., Pérez-Ramírez, D., Sollerman, J., et al. 2005, A\&A, 444, 71

Gullberg, B., De Breuck, C., Vieira, J. D., et al. 2015, MNRAS, 449, 2883

Guo, Y., Rafelski, M., Faber, S. M., et al. 2016, ApJ, submitted [arXiv: 1604.05314]

Güsten, R., Nyman, L. Å., Schilke, P., et al. 2006, A\&A, 454, L13

Habing, H. J. 1968, Bull. Astron. Inst. Netherlands, 19, 421

Hammer, F., Flores, H., Schaerer, D., et al. 2006, A\&A, 454, 103

Han, X. H., Hammer, F., Liang, Y. C., et al. 2010, A\&A, 514, A24

Hashimoto, T., Perley, D. A., Ohta, K., et al. 2015, ApJ, 806, 250
Hatsukade, B., Kohno, K., Endo, A., et al. 2007, PASJ, 59, 67

Hatsukade, B., Ohta, K., Endo, A., et al. 2014, Nature, 510, 247

Herrera-Camus, R., Bolatto, A. D., Wolfire, M. G., et al. 2015, ApJ, 800, 1

Hjorth, J., \& Bloom, J. S. 2012, in Gamma Ray Burst (Cambridge University Press), 169

Hjorth, J., Sollerman, J., Møller, P., et al. 2003, Nature, 423, 847

Hjorth, J., Malesani, D., Jakobsson, P., et al. 2012, ApJ, 756, 187

Hu, C. Y., Naab, T., Walch, S., Glover, S. C. O., \& Clark, P. C. 2016, MNRAS, 458, 3528

Hughes, T. M., Ibar, E., Villanueva, V., et al. 2016, A\&A, submitted

Hunt, L. K., Palazzi, E., Michałowski, M. J., et al. 2014a, A\&A, 565, A112

Hunt, L. K., Testi, L., Casasola, V., et al. 2014b, A\&A, 561, A49

Hunt, L. K., García-Burillo, S., Casasola, V., et al. 2015, A\&A, 583, A114

Iglesias-Páramo, J., Boselli, A., Gavazzi, G., \& Zaccardo, A. 2004, A\&A, 421, 887

Iglesias-Páramo, J., Buat, V., Hernández-Fernández, J., et al. 2007, ApJ, 670, 279

Israel, F. P., Maloney, P. R., Geis, N., et al. 1996, ApJ, 465, 738

Izzard, R. G., Ramirez-Ruiz, E., \& Tout, C. A. 2004, MNRAS, 348, 1215

Jakobsson, P., Hjorth, J., Malesani, D., et al. 2012, ApJ, 752, 62

Japelj, J., Vergani, S. D., Salvaterra, R., et al. 2016, A\&A, 590, A129

Joye, W. A., \& Mandel, E. 2003, in Astronomical Data Analysis Software and

Systems XII, eds. H. E. Payne, R. I. Jedrzejewski, \& R. N. Hook, ASP Conf. Ser., 295, 489

Kaufman, M. J., Wolfire, M. G., \& Hollenbach, D. J. 2006, ApJ, 644, 283

Kennicutt, R. C. 1998, ARA\&A, 36, 189

Kewley, L. J., \& Dopita, M. A. 2002, ApJS, 142, 35

Kewley, L. J., Geller, M. J., \& Jansen, R. A. 2004, AJ, 127, 2002

Krühler, T., Fynbo, J. P. U., Geier, S., et al. 2012, A\&A, 546, A8

Krühler, T., Ledoux, C., Fynbo, J. P. U., et al. 2013, A\&A, 557, A18

Krumholz, M. R. 2012, ApJ, 759, 9

Le Floc'h, E., Charmandaris, V., Forrest, W. J., et al. 2006, ApJ, 642, 636

Lee, J. C., Gil de Paz, A., Tremonti, C., et al. 2009, ApJ, 706, 599

Levesque, E. M., Berger, E., Kewley, L. J., \& Bagley, M. M. 2010a, AJ, 139, 694

Levesque, E. M., Kewley, L. J., Graham, J. F., \& Fruchter, A. S. 2010b, ApJ, 712, L26

Madden, S. C. 2000, New Astron., 44, 249

Madden, S. C., Poglitsch, A., Geis, N., Stacey, G. J., \& Townes, C. H. 1997, ApJ, 483, 200

Madden, S. C., Rémy-Ruyer, A., Galametz, M., et al. 2013, PASP, 125, 600

Maiolino, R., Nagao, T., Grazian, A., et al. 2008, A\&A, 488, 463

Malhotra, S., Kaufman, M. J., Hollenbach, D., et al. 2001, ApJ, 561, 766

Martin, D. C., Chang, D., Matuszewski, M., et al. 2014, ApJ, 786, 106

McMullin, J. P., Waters, B., Schiebel, D., Young, W., \& Golap, K. 2007, in Astronomical Data Analysis, eds. R. A. Shaw, F. Hill, \& D. J. Bell, Software and Systems XVI, ASP Conf. Ser., 376, 127

Meurer, G. R., Wong, O. I., Kim, J. H., et al. 2009, ApJ, 695, 765

Michałowski, M. J., Hjorth, J., Castro Cerón, J. M., \& Watson, D. 2008, ApJ, 672,817

Michałowski, M. J., Hjorth, J., Malesani, D., et al. 2009, ApJ, 693, 347

Michałowski, M. J., Hjorth, J., \& Watson, D. 2010a, A\&A, 514, A67

Michałowski, M. J., Watson, D., \& Hjorth, J. 2010b, ApJ, 712, 942

Michałowski, M. J., Dunlop, J. S., Cirasuolo, M., et al. 2012a, A\&A, 541, A85

Michałowski, M. J., Kamble, A., Hjorth, J., et al. 2012b, ApJ, 755, 85

Michałowski, M. J., Hayward, C. C., Dunlop, J. S., et al. 2014a, A\&A, 571, A75

Michałowski, M. J., Hunt, L. K., Palazzi, E., et al. 2014b, A\&A, 562, A70

Michałowski, M. J., Gentile, G., Hjorth, J., et al. 2015, A\&A, 582, A78

Mitchell, P. D., Lacey, C. G., Baugh, C. M., \& Cole, S. 2013, MNRAS, 435, 87

Modjaz, M., Kewley, L., Kirshner, R. P., et al. 2008, AJ, 135, 1136

Murphy, E. J., Condon, J. J., Schinnerer, E., et al. 2011, ApJ, 737, 67

Neri, R., Downes, D., Cox, P., \& Walter, F. 2014, A\&A, 562, A35

Östlin, G., Zackrisson, E., Sollerman, J., Mattila, S., \& Hayes, M. 2008, MNRAS, 387, 1227

Ott, S. 2010, in Astronomical Data Analysis Software and Systems XIX, eds. Y Mizumoto, K. I. Morita, M. Ohishi, ASP Conf. Ser., 434, 139

Pacifici, C., Charlot, S., Blaizot, J., \& Brinchmann, J. 2012, MNRAS, 421, 2002

Pagel, B. E. J., Edmunds, M. G., Blackwell, D. E., Chun, M. S., \& Smith, G. 1979, MNRAS, 189, 95

Perley, D. A., \& Perley, R. A. 2013, ApJ, 778, 172

Perley, D. A., Levan, A. J., Tanvir, N. R., et al. 2013, ApJ, 778, 128

Perley, D. A., Perley, R. A., Hjorth, J., et al. 2015, ApJ, 801, 102

Perley, D. A., Tanvir, N. R., Hjorth, J., et al. 2016, ApJ, 817, 8

Petrovic, J., Langer, N., Yoon, S. C., \& Heger, A. 2005, A\&A, 435, 247

Pettini, M., \& Pagel, B. E. J. 2004, MNRAS, 348, L59

Pety, J. 2005, in SF2A-2005: Semaine de l'Astrophysique Francaise, eds. F. Casoli, T. Contini, J. M. Hameury, \& L. Pagani, 721

Pilbratt, G. L., Riedinger, J. R., Passvogel, T., et al. 2010, A\&A, 518, L1 
Piranomonte, S., Japelj, J., Vergani, S. D., et al. 2015, MNRAS, 452, 3293

Podsiadlowski, P., Mazzali, P. A., Nomoto, K., Lazzati, D., \& Cappellaro, E. 2004, ApJ, 607, L17

Podsiadlowski, P., Ivanova, N., Justham, S., \& Rappaport, S. 2010, MNRAS, 406, 840

Poglitsch, A., Krabbe, A., Madden, S. C., et al. 1995, ApJ, 454, 293

Poglitsch, A., Waelkens, C., Geis, N., et al. 2010, A\&A, 518, L2

Pound, M. W., \& Wolfire, M. G. 2008, in Astronomical Data Analysis Software and Systems XVII, eds. R. W. Argyle, P. S. Bunclark, J. R. Lewis, ASP Conf. Ser., 394, 654

Price, P. A., Kulkarni, S. R., Berger, E., et al. 2002, ApJ, 571, L121

Prochaska, J. X., Bloom, J. S., Chen, H. W., et al. 2004, ApJ, 611, 200

Prochaska, J. X., Sheffer, Y., Perley, D. A., et al. 2009, ApJ, 691, L27

Rafelski, M., Gardner, J. P., Fumagalli, M., et al. 2016, ApJ, 825, 87

Rauch, M., Becker, G. D., \& Haehnelt, M. G. 2016, MNRAS, 455, 3991

Ribaudo, J., Lehner, N., Howk, J. C., et al. 2011, ApJ, 743, 207

Rigopoulou, D., Hopwood, R., Magdis, G. E., et al. 2014, ApJ, 781, L15

Rubin, D., Hony, S., Madden, S. C., et al. 2009, A\&A, 494, 647

Salpeter, E. E. 1955, ApJ, 121, 161

Sánchez Almeida, J., Muñoz-Tuñón, C., Elmegreen, D. M., Elmegreen, B. G., \& Méndez-Abreu, J. 2013, ApJ, 767, 74

Sánchez Almeida, J., Elmegreen, B. G., Muñoz-Tuñón, C., \& Elmegreen, D. M. 2014a, A\&ARv, 22, 71

Sánchez Almeida, J., Morales-Luis, A. B., Muñoz-Tuñón, C., et al. 2014b, ApJ, 783,45

Sancisi, R., Fraternali, F., Oosterloo, T., \& van der Hulst, T. 2008, A\&ARv, 15, 189

Sargsyan, L., Lebouteiller, V., Weedman, D., et al. 2012, ApJ, 755, 171

Savaglio, S., Glazebrook, K., \& LeBorgne, D. 2009, ApJ, 691, 182

Savaglio, S., Rau, A., Greiner, J., et al. 2012, MNRAS, 420, 627

Schady, P., Savaglio, S., Müller, T., et al. 2014, A\&A, 570, A52

Schady, P., Krühler, T., Greiner, J., et al. 2015, A\&A, 579, A126

Schaye, J., Dalla Vecchia, C., Booth, C. M., et al. 2010, MNRAS, 402, 1536

Schulze, S., Malesani, D., Cucchiara, A., et al. 2014, A\&A, 566, A102

Schulze, S., Chapman, R., Hjorth, J., et al. 2015, ApJ, 808, 73

Silva, L., Granato, G. L., Bressan, A., \& Danese, L. 1998, ApJ, 509, 103

Simha, V., Weinberg, D. H., Conroy, C., et al. 2014, MNRAS, submitted [arXiv: 1404.0402]

Sollerman, J., Östlin, G., Fynbo, J. P. U., et al. 2005, New Astron., 11, 103

Sollerman, J., Jaunsen, A. O., Fynbo, J. P. U., et al. 2006, A\&A, 454, 503

Solomon, P. M., Downes, D., Radford, S. J. E., \& Barrett, J. W. 1997, ApJ, 478, 144

Stacey, G. J., Geis, N., Genzel, R., et al. 1991, ApJ, 373, 423

Stacey, G. J., Hailey-Dunsheath, S., Ferkinhoff, C., et al. 2010, ApJ, 724, 957

Stanek, K. Z., Matheson, T., Garnavich, P. M., et al. 2003, ApJ, 591, L17

Stanway, E. R., Davies, L. J. M., \& Levan, A. J. 2010, MNRAS, 409, L74

Stanway, E. R., Levan, A. J., Tanvir, N., et al. 2015a, MNRAS, 446, 3911

Stanway, E. R., Levan, A. J., Tanvir, N. R., Wiersema, K., \& van der Laan,

T. P. R. 2015b, ApJ, 798, L7

Stott, J. P., Sobral, D., Bower, R., et al. 2013, MNRAS, 436, 1130

Sullivan, M., Treyer, M. A., Ellis, R. S., et al. 2000, MNRAS, 312, 442

Swinbank, A. M., Karim, A., Smail, I., et al. 2012, MNRAS, 427, 1066

Thöne, C. C., Fynbo, J. P. U., Östlin, G., et al. 2008, ApJ, 676, 1151

Tinney, C., Stathakis, R., Cannon, R., et al. 1998, IAU Circ., 6896

Trenti, M., Perna, R., \& Jimenez, R. 2015, ApJ, 802, 103

Tumlinson, J., Prochaska, J. X., Chen, H. W., Dessauges-Zavadsky, M., \& Bloom J. S. 2007, ApJ, 668, 667

Turner, J. L., Beck, S. C., Benford, D. J., et al. 2015, Nature, 519, 331 van den Heuvel, E. P. J., \& Portegies Zwart, S. F. 2013, ApJ, 779, 114

Vassilev, V., Meledin, D., Lapkin, I., et al. 2008, A\&A, 490, 1157

Vergani, S. D., Salvaterra, R., Japelj, J., et al. 2015, A\&A, 581, A102

Vreeswijk, P. M., Ellison, S. L., Ledoux, C., et al. 2004, A\&A, 419, 927

Wang, J., Serra, P., Józsa, G. I. G., et al. 2015, MNRAS, 453, 2399
Wang, L., Norberg, P., Gunawardhana, M. L. P., et al. 2016, MNRAS, 461, 1898

Watson, D., French, J., Christensen, L., et al. 2011, ApJ, 741, 58

Weisz, D. R., Johnson, B. D., Johnson, L. C., et al. 2012, ApJ, 744, 44

Wijesinghe, D. B., da Cunha, E., Hopkins, A. M., et al. 2011, MNRAS, 415, 1002

Wolfire, M. G., Hollenbach, D., \& Tielens, A. G. G. M. 1989, ApJ, 344, 770

Woosley, S. E., \& Heger, A. 2006, ApJ, 637, 914

Yoon, S. C., \& Langer, N. 2005, A\&A, 443, 643

Yoon, S. C., Langer, N., \& Norman, C. 2006, A\&A, 460, 199

1 Scottish Universities Physics Alliance (SUPA), Institute for Astronomy, University of Edinburgh, Royal Observatory, Blakford Hill, Edinburgh, EH9 3HJ, UK

e-mail: mm@roe.ac.uk

2 Herschel Science Centre (ESA-ESAC), 28.692 Villanueva de la Cañada, Madrid, Spain

3 Dark Cosmology Centre, Niels Bohr Institute, University of Copenhagen, Juliane Maries Vej 30, 2100 Copenhagen Ø, Denmark

4 Centre for Extragalactic Astronomy, Department of Physics, Durham University, South Road, Durham DH13LE, UK

5 Centre for Astronomy, Nicolaus Copernicus University, Faculty of Physics, Astronomy and Informatics, Grudziadzka 5, 87100 Toruń, Poland

6 Astronomical Observatory, Adam Mickiewicz University, Słoneczna 36, 60-268, Poznań, Poland

7 Centro de Astronomia e Astrofísica da Universidade de Lisboa, Observatório Astronómico de Lisboa, Tapada da Ajuda, 1349-018 Lisbon, Portugal

8 Leiden Observatory, Leiden University, PO Box 9513, 2300 RA Leiden, The Netherlands

9 INAF-Osservatorio Astrofisico di Arcetri, Largo E. Fermi 5, 50125 Firenze, Italy

10 Sterrenkundig Observatorium, Universiteit Gent, Krijgslaan 281-S9, 9000 Gent, Belgium

11 Instituto de Astrofísica de Andalucía (IAA-CSIC), Glorieta de la Astronomía s/n, 18.008 Granada, Spain

12 Department of Physics and Astrophysics, Vrije Universiteit Brussel, Pleinlaan 2, 1050 Brussels, Belgium

13 Laboratoire AIM-Paris-Saclay, CEA/DSM/Irfu - CNRS Université Paris Diderot, CE-Saclay, pt courrier 131, 91191 Gif-sur-Yvette, France

14 XMM Science Operation Centre, ESAC/ESA, Villafranca del Castillo, 28692 Madrid, Spain

15 Thüringer Landessternwarte Tautenburg, Sternwarte 5, 07778 Tautenburg, Germany

16 Technical University of Denmark, Department of Physics, Fysikvej, building 309, 2800 Kgs. Lyngby, Denmark

17 Centro de Astrobiología (INTA-CSIC), Ctra. M-108, km 4, 28850 Torrejón de Ardoz, Madrid, Spain

18 INAF-IASF Bologna, via Gobetti 101, 40129 Bologna, Italy

19 Max-Planck-Institut für Extraterrestrische Physik, Giessenbachstraße, 85748 Garching, Germany

20 The Oskar Klein Centre, Department of Astronomy, AlbaNova, Stockholm University, 10691 Stockholm, Sweden

21 Key Laboratory of Space Astronomy and Technology, National Astronomical Observatories, Chinese Academy of Sciences, 20A Datun Road, Beijing 100012, PR China 


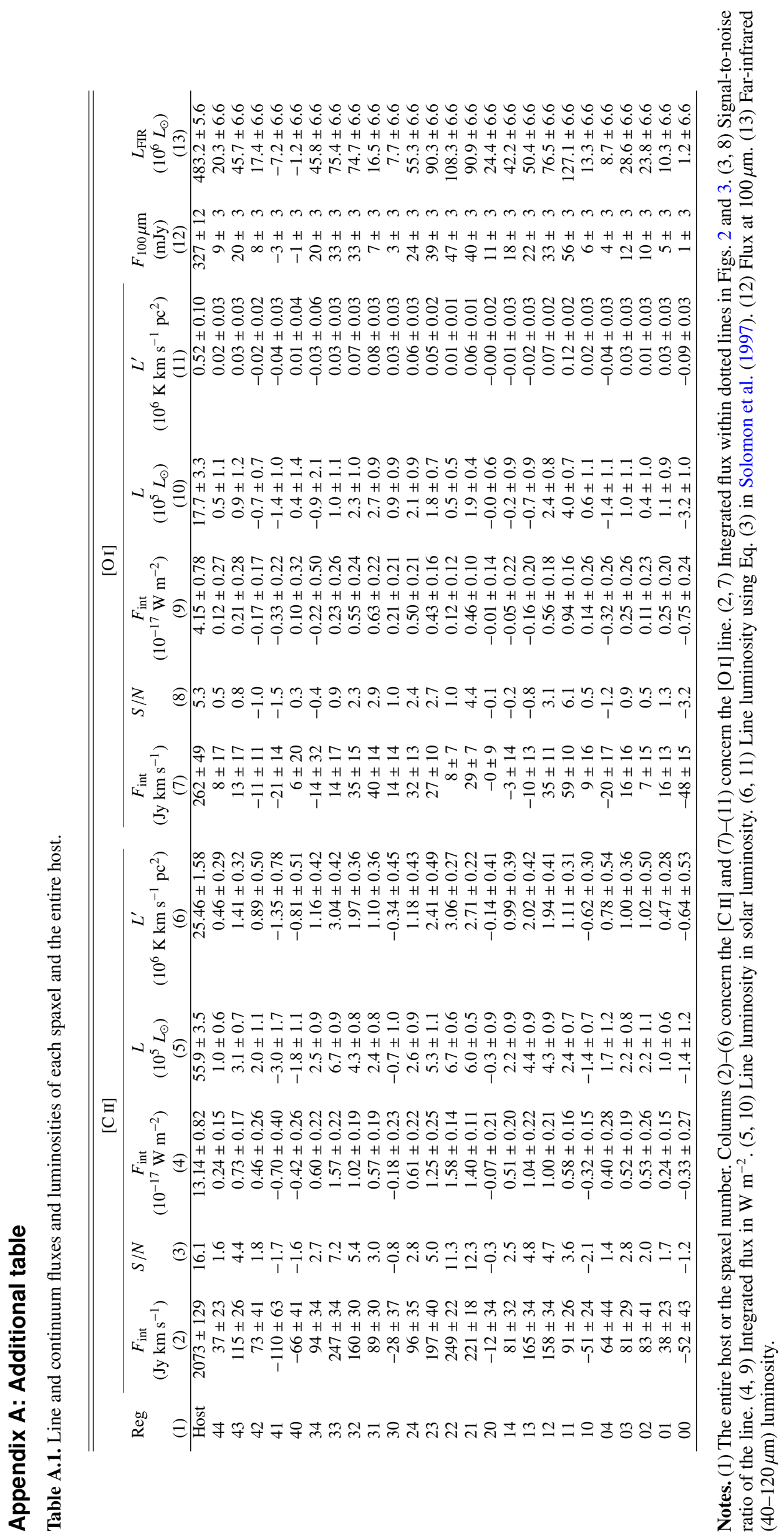

